Convergence analysis of an algorithm for accurate inverse Cholesky factorization

\author{
高精度逆コレスキー分解アルゴリズムの \\ 収束解析に関する研究
}

February 2016

Yuka YANAGISAWA

柳澤 優香 


\title{
Convergence ANALYSIS OF AN ALGORITHM FOR ACCURATE INVERSE CHOLESKY FACTORIZATION 高精度逆コレスキー分解アルゴリズムの 収束解析に関する研究
}

February 2016

\author{
Waseda University \\ Graduate School of Fundamental Science and Engineering \\ Department of Pure and Applied Mathematics \\ Research on Numerical Analysis
}

YUKA YANAGISAWA 
(C) Copyright by Yuka Yanagisawa, 2016

All Rights Reserved. 


\section{ACKNOWLEDGMENTS}

First and foremost, the author would like to express her largest gratitude to her supervisor Professor Shin'ichi Oishi, for his support and encouragement during her doctoral degree in his laboratory. Without his guidance and reassuring support, this thesis would not have been possible.

The author also express my sincere gratitude to Professor Daisuke Takahashi and Professor Masahide Kashiwagi for valuable comments as the second reader.

The author would particularly like to appreciate Associate Professor Takeshi Ogita at Tokyo Woman's Christian University for his encouragement and helpful advices.

The author wishes to thank to Associate Professor Katsuhisa Ozaki at Shibaura Institute of Technology for his kind support.

Finally, the author is grateful to all members of Oishi Laboratory in Waseda University.

Yuka Yanagisawa 



\section{Contents}

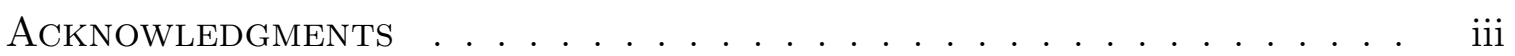

Chapter 1. Introduction . . . . . . . . . . . . . . . . . 1

Chapter 2. Preliminaries . . . . . . . . . . . . . . . . . 7

2.1. Notation and definitions . . . . . . . . . . . . . . . . 8

2.2. Accurate matrix multiplication . . . . . . . . . . . . . 9

2.3. Accurate and robust inverse Cholesky factorization . . . . . . . . . 15

Chapter 3. Convergence Analysis of An Algorithm for ACCurate

inverse Cholesky factorizhtion . . . . . . . . . . . . . . . . . . . . 21

3.1. Analysis ........................ . . . 22

3.2. Numerical experiments . . . . . . . . . . . . . . . . 36

Chapter 4. A modified Algorithm for aCCurate inverse Cholesky

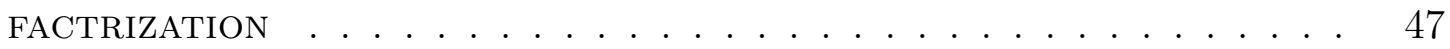

4.1. Proposed algorithm . . . . . . . . . . . . . . . . 48

4.2. Analysis of accuracy on $X \ldots \ldots \ldots$. . . . . . . . . . 52

4.3. Numerical experiments . . . . . . . . . . . . . . . . . 58

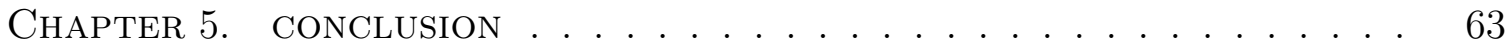
ApPENDIX:

Appendix A. Executable Matlab code . . . . . . . . . . . . 65

BIBLIOGRAPHY . . . . . . . . . . . . . . . . . . 77 
Chapter 1

\section{INTRODUCTION}


Let $\mathbb{R}$ be the set of real numbers, and $\mathbb{F}$ be a set of floating-point numbers conforming to IEEE standard 754. The relative rounding error unit of floating-point arithmetic is denoted by $\mathbf{u}$. In binary64 (double precision) arithmetic, $\mathbf{u}=2^{-53} \approx$ $1.1 \times 10^{-16}$. Throughout this paper, we assume that neither overflow nor underflow occurs. For $A \in \mathbb{R}^{n \times n}$ define $\kappa(A):=\|A\| \cdot\left\|A^{-1}\right\|$ as the condition number of $A$, where $\|\cdot\|$ stands for spectral norm for matrices and Euclidean norm for vectors.

In this thesis, we consider to treat the case where $A$ is symmetric, positive definite and extremely ill-conditioned such that

$$
\kappa(A)>\mathbf{u}^{-1}
$$

This means no correct digit can be expected in an approximate solution $\tilde{x}$ when solving a linear system $A x=b$ in working precision $\mathbf{u}$. There are several applications in which the case (1) appears, for example, ill-conditioned Gramian matrices deriving from discretization of partial differential equations, Cholesky QR method [20] and so forth.

In about 1990, Rump [12] showed that a approximate inverse of extremely illconditioned matrix may still be useful as a preconditioner. He derived a method for inverting an extremely ill-conditioned matrix which requires the possibility to calculate a dot product in higher precision. Fortunately, Ogita et al. [8] developed a method for calculating a dot product in $k$-fold precision in 2005. In the same year, Ohta et al. [9] redeveloped Rump's method using the accurate dot product algorithm [8]. However, why Rump's method is so efficient for ill-conditioned matrices had not yet been known. In 2007, Oishi et al. [10] presented a proof why a modified version of the Rump's method works so well. In 2009, Rump [16] showed the mechanism of his method by himself. In 2010, Ogita [6] proposed a framework with the same spirit of Rump's method for an accurate and efficient computation of matrix factorizations such as inverse LU and inverse QR factorizations. He analyzed these algorithms in 
detail and confirmed that it coincides with numerical results. Nevertheless, it is not possible to adapt his proposed framework directly to Cholesky factorization of $A$. Because a floating-point Cholesky factorization of an ill-conditioned matrix such that (1) may break down by the presence of an imaginary root due to the accumulation of rounding errors, even if the matrix is symmetric and positive definite. In 2012, Ogita and Oishi [7] derived an iterative algorithm to calculate a good approximate inverse of the exact Cholesky factor. To avoid the break-down, a diagonal shift is applied to $A$ in the Ogita-Oishi's algorithm. As is the case with $[\mathbf{6}, \mathbf{9}]$, the OgitaOishi's algorithm is needed an algorithm for accurately computing a dot product as if computed in $k$-fold working precision and rounded into the sum of $l$ pieces of working precision floating-point numbers for $k \geq 2$ and $1 \leq l \leq k$. Fortunately, such accurate dot product algorithms have been proposed in $[\mathbf{8}, \mathbf{1 1}, \mathbf{1 6}, \mathbf{1 8}, \mathbf{1 9}]$, and they are very fast. By numerical results in [7], the performance of this algorithm was evaluated. However, the Ogita-Oishi's algorithm has not been analyzed in detail and explained reasons for convergency.

This thesis has two purposes. The first is to analyze the behavior of the algorithm in detail and explain its convergency by the use of numerical error analysis. Main analysis to be explained is that each iteration of the Ogita-Oishi's algorithm reduces the condition number of a preconditioned matrix by a factor around $n^{2} \mathbf{u}$ until convergence, which is consistent with the numerical results. As is the case with $[\mathbf{1 6}]$, we do not give a completely rigorous proof but arguments how and why the algorithm works. The key of the analysis is in multiplicative corrections of a sum of $m$ matrices $X=\sum_{i=1}^{m} X_{i}, X_{i} \in \mathbb{F}^{n \times n}$, which is an accurate inverse of $\hat{R}$ such that $A=\hat{R}^{\top} \hat{R}$ satisfying $\kappa\left(X^{\top} A X\right)=1$. Note that $\hat{R}$ is upper triangular. The Ogita-Oishi's algorithm requires an algorithm for accurate dot product, which helps to treat extremely ill-conditioned matrices. We show that it is important to choose adequate computational precision for calculating dot products in the algorithm, which is sufficient for 
convergence and almost optimal in terms of computational effort. As we will see this does influence the analysis. We justify the optimality of high precision arithmetic for dot product used in the Ogita-Oishi's algorithm by our numerical error analysis. This analysis is a vastly extended version of [22].

The second is to propose a modified version of the Ogita-Oishi's algorithm for calculating a more accurate inverse $X$ of $\hat{R}$ than the original one. Namely, the proposed algorithm provides $X$ satisfying

$$
\left\|I-X^{\top} A X\right\| \approx \mathbf{u}
$$

In this thesis, we use the " $\approx$ " notation to indicate that two quantities are approximately equal. For example, for $x, y \in \mathbb{R}, x \approx y$ means that $|x|=\varphi \cdot|y|$ for reasonably small constant $\varphi$. As mentioned before, for the case (1), a floating-point Cholesky factorization may break down due to the accumulation of rounding errors. To avoid the break-down, a diagonal shift is applied to $A$ in the Ogita-Oishi's algorithm. As we will see from some numerical results in Chapter 3, the Ogita-Oishi's algorithm gives an approximate inverse $X$ satisfying

$$
n^{2} \mathbf{u} \approx\left\|I-X^{\top} A X\right\|<1
$$

The diagonal shift is the reason why $n^{2} \mathbf{u}$ is the lower bound. To overcome it, we find the condition such that Cholesky factorization of a preconditioned matrix never breaks down without the diagonal shift.

The rest of the thesis is organized as follows: in the following chapter, we explain some basic definitions, and we introduce an algorithm for accurately computing a dot product. Moreover, we state the algorithm [7] for an accurate inverse Cholesky factorization. In Chapter 3, we present detailed analysis of the algorithm. Furthermore, we present some numerical results for confirming our analysis and comparing the algorithm with a standard Cholesky factorization by using long precision arithmetic 
$[2,5]$, in terms of measured computing time for verifying the positive definiteness of an input matrix. In Chapter 4, we propose a modified algorithm for an accurate inverse Cholesky factorization. Moreover, we present some numerical results for comparing the proposed algorithm with the original one in terms of both the accuracy on $X$ and measured computing time for convergence. Finally, we conclude our results in Chapter 5. In addition, we give the complete Matlab-code for our algorithms. 

Chapter 2

\section{Preliminaries}


This section is structured as follows. In Section 2.1, we prepare some notations, definitions and error estimations of floating point arithmetic. In Section 2.2, we describe an algorithm of accurate matrix multiplication $[\mathbf{1 1}, \mathbf{1 6}]$. In Section 2.3, we introduce the Ogita-Oishi algorithm [7] for an accurate inverse Cholesky factorization.

\subsection{Notation AND DEFinitions}

For $A=\left(a_{i j}\right), B=\left(b_{i j}\right) \in \mathbb{R}^{n \times n},|A|=\left(\left|a_{i j}\right|\right) \in \mathbb{R}^{n \times n}$ denotes a nonnegative matrix consisting of entrywise absolute values, and an inequality $A \leq B$ is understood entrywise, i.e., $a_{i j} \leq b_{i j}$ for all $(i, j)$. The notation $A \geq O$ means that all elements of $A$ are nonnegative. Similar notation applies to real vectors. Let $I$ denote the identity matrix. For readability $\varphi(\gamma)$ denotes a constant such as $\varphi(\gamma)=c \cdot \gamma$ for factor $c$ near 1.

The eigenvalues of $A$ is denoted by $\lambda_{i}(A)$ with $\lambda_{1}(A) \geq \lambda_{2}(A) \geq \cdots \geq \lambda_{n}(A)$. The trace of $A=\left(a_{i j}\right) \in \mathbb{R}^{n \times n}$ is defined by

$$
\operatorname{tr}(A):=\sum_{i=1}^{n} a_{i i}=\sum_{i=1}^{n} \lambda_{i}(A)
$$

Computing an approximate inverse of an upper triangular matrix $R \in \mathbb{F}^{n \times n}$ is denoted by

$$
T:=\operatorname{triinv}(R)
$$

which solves a matrix equation $R T=I$ for $T$ using a standard numerical algorithm (e.g., xTRSV in BLAS and xTRTRI in LAPACK) in working precision such as binary64.

The sum of the absolute values of off-diagonal elements in the $i$-th row of $A$ is denoted by

$$
r_{i}(A)=\sum_{i \neq j}\left|a_{i j}\right|
$$

We assume that the following three types of rounding modes are defined in IEEE standard 754: Let $c \in \mathbb{R}$. 
(1) Rounding to nearest even (default): Round $c$ to the nearest floating point number $\tilde{f} \in \mathbb{F}$ satisfying $|\tilde{f}-c|=\min _{f \in \mathbb{F}}|f-c|$.

(2) Rounding upwards: Round $c$ to the smallest floating point number $\bar{f} \in \mathbb{F}$ satisfying $\bar{f} \geq c$.

(3) Rounding downwards: Round $c$ to the largest floating point number $f \in \mathbb{F}$ satisfying $\underline{f} \leq c$.

Let $f(\cdot), f l_{\triangle}(\cdot)$ and $f l_{\nabla}(\cdot)$ denote that an expression inside the parentheses is evaluated by floating-point arithmetic in rounding to nearest, rounding upwards and rounding downwards, respectively. We assume the use of the following standard model: For $a, b \in \mathbb{F}$ and $\circ \in\{+,-, *, /\}$ it holds that

$$
f(a \circ b)=(1+\varepsilon)(a \circ b), \quad|\varepsilon| \leq \mathbf{u} .
$$

For $0 \leq a \in \mathbb{F}$,

$$
f\left(a^{\frac{1}{2}}\right)=a^{\frac{1}{2}}\left(1+\varepsilon_{1}\right), \quad\left|\varepsilon_{1}\right| \leq \mathbf{u} .
$$

Let $\mathbb{F}_{[l]}$ be a set of sum of floating-point numbers such that

$$
\mathbb{F}_{[l]}:=\left\{x \in \mathbb{R}: x=\sum_{i=1}^{l} x_{i}, x_{i} \in \mathbb{F}, l \in \mathbb{N}\right\} .
$$

Note that $\mathbb{F}=\mathbb{F}_{[1]} \subseteq \mathbb{F}_{[l]} \subset \mathbb{R}$.

\subsection{ACCurate matrix multiplication}

The Ogita-Oishi's algorithm [7] requires an algorithm for accurate dot product as if calculated in $k$-fold precision, where the result is stored in $l$ terms. In this paper we need only these two cases $l=1$ and $l=k$. Fortunately we have a function of calculating $C_{1: l} \in \mathbb{F}_{[l]}^{n \times n}$ for any $k, l \in \mathbb{N}, l \leq k$ satisfying

$$
\left|A B-C_{1: l}\right| \leq \varphi\left(\mathbf{u}^{l}\right)|A B|+\varphi\left(\mathbf{u}^{k}\right)|A||B| .
$$


Note that

$$
C_{1: l}=\sum_{i=1}^{l} C_{i} \quad \text { with } C_{i} \in \mathbb{F}^{n \times n}, i=1,2, \ldots, l .
$$

Namely, $C_{1: l}$ is an approximation of $A B$ as if computed in $k$-fold working precision and rounded into $l$ pieces of working precision floating-point numbers. We write such a function as

$$
C_{1: l}:=\{A B\}_{k}^{l}
$$

which provides $C_{1: l}$ satisfying (4).

Let $\left\langle A_{M}, A_{R}\right\rangle$ denote an interval matrix of the midpoint-radius representation such that

$$
\left\langle A_{M}, A_{R}\right\rangle:=\left\{X \in \mathbb{R}^{n \times n}:\left|X-A_{M}\right| \leq A_{R}\right\}
$$

with a midpoint $A_{M} \in \mathbb{F}^{n \times n}$ and a radius $A_{R} \in \mathbb{F}^{n \times n}, A_{R} \geq O$. Similar notation in (5) applies to $B^{\top} A B$, i.e.,

$$
\langle G, E\rangle:=\left\{B^{\top} A B\right\}_{k}^{1}
$$

which provides $G=G^{\top}, E \in \mathbb{F}^{n \times n}$ satisfying $B^{\top} A B \in\langle G, E\rangle$. Then we require

$$
\left|B^{\top} A B-G\right| \approx \varphi(\mathbf{u})\left|B^{\top} A B\right|+\varphi\left(\mathbf{u}^{k}\right)\left|B^{\top}\right||A||B|=: E
$$

REMARK 1. For (6), in practice, it can be realized by

$$
\left\langle C_{1: k}, E_{1}\right\rangle:=\{A B\}_{k}^{k} \text { and }\left\langle D, E_{2}\right\rangle:=\left\{B^{\top} C_{1: k}\right\}_{k}^{1}
$$

which satisfy

$$
\begin{aligned}
& \left|A B-C_{1: k}\right| \leq \varphi\left(\mathbf{u}^{k}\right)|A B|+\varphi\left(\mathbf{u}^{k}\right)|A||B|=: E_{1} \\
& \left|B^{\top} C_{1: k}-D\right| \leq \varphi(\mathbf{u})\left|B^{\top} C_{1: k}\right|+\varphi\left(\mathbf{u}^{k}\right)\left|B^{\top}\right|\left|C_{1: k}\right|=: E_{2}
\end{aligned}
$$


where $C_{1: k} \in \mathbb{F}_{[k]}^{n \times n}, D, E_{1}, E_{2} \in \mathbb{F}^{n \times n}$. From (7) and (8), it holds that

$$
\begin{aligned}
\left|B^{\top} A B-D\right| & \leq\left|B^{\top} A B-B^{\top} C_{1: k}\right|+\left|B^{\top} C_{1: k}-D\right| \\
& \leq\left|B^{\top}\right|\left|A B-C_{1: k}\right|+\left|B^{\top} C_{1: k}-D\right| \\
& \leq\left|B^{\top}\right| E_{1}+E_{2}
\end{aligned}
$$

and

$$
\begin{aligned}
E_{2}= & \varphi(\mathbf{u})\left(\left|B^{\top} A B\right|+\left|B^{\top}\right| E_{1}\right)+\varphi\left(\mathbf{u}^{k}\right)\left|B^{\top}\right|\left(|A B|+E_{1}\right) \\
\approx & \varphi(\mathbf{u})\left|B^{\top} A B\right|+\varphi\left(\mathbf{u}^{k+1}\right)\left|B^{\top}\right||A||B| \\
& \quad+\varphi\left(\mathbf{u}^{k}\right)\left|B^{\top}\right||A B|+\varphi\left(\mathbf{u}^{2 k}\right)\left|B^{\top}\right||A||B| \\
\approx & \varphi(\mathbf{u})\left|B^{\top} A B\right|+\varphi\left(\mathbf{u}^{k}\right)\left|B^{\top}\right||A||B| .
\end{aligned}
$$

Inserting (7) and (10) into (9) implies

$$
\left|B^{\top} A B-D\right| \approx \varphi(\mathbf{u})\left|B^{\top} A B\right|+\varphi\left(\mathbf{u}^{k}\right)\left|B^{\top}\right||A||B| .
$$

Such accurate dot product algorithms satisfying (4) have been proposed in $[\mathbf{8}, \mathbf{1 8}$, 19]. In this thesis, we use error-free transformation algorithm of matrix multiplication by fast routines of matrix multiplication which have been developed in [11]. After this transformation, we apply an accurate summation algorithm $[\mathbf{8}, \mathbf{1 6}]$ for floating-point numbers.

We describe an algorithm for error-free transformation of the matrix $A \in \mathbb{F}^{m \times n}$ without rounding errors. 
Algorithm 2.1 (Ozaki [11]). This algorithm transforms $A$ into $\sum_{i=1}^{h_{A}} D_{i}$ without rounding errors.

$$
\begin{aligned}
& \text { function } D_{1: h_{A}}=\text { Ozaki_Split }(A) \\
& n=\operatorname{size}(A, 2) \text {; } \\
& \mu=\max (\operatorname{abs}(A),[], 2) ; \quad \% \mu \in \mathbb{F}^{m \times 1} \text { with } \mu(i)=\max _{1 \leq j \leq q}\left|a_{i j}\right| \text {; } \\
& \alpha=\mathrm{fl}\left(\left\lfloor-\frac{\log _{2}(\mathbf{u})+\left\lceil\log _{2}(n)\right\rceil}{2}\right\rfloor\right) ; \\
& \beta=\mathrm{fl}\left(\left\lceil\frac{-\log _{2}(\mathbf{u})+\log _{2}(n)}{2}\right\rceil\right) \text {; } \\
& \omega=\mathrm{fl}\left(2^{\left\lceil\left(\log _{2}(\mu)\right\rceil+\beta\right)}\right) \text {; } \\
& S=\operatorname{repmat}(\omega, n) ; \quad \% S=\omega \cdot e^{\top} \text { for } e=(1,1 \ldots 1)^{\top} \in \mathbb{F}^{n} \\
& h_{A}=1 \text {; }
\end{aligned}
$$

While any $(\operatorname{any}(A)) \quad \%$ Determine if any array elements are nonzero

$$
\begin{aligned}
& D_{h_{A}}=\mathrm{fl}((A+S)-S) ; \\
& A=\mathrm{fl}\left(A-D_{h_{A}}\right) ; \\
& S=\alpha A ; \\
& h_{A}=h_{A}+1 ;
\end{aligned}
$$

Similarly, the matrix $B$ is split into $\sum_{i=1}^{h_{B}} E_{i}$ by $E=$ Ozaki_Split $\left(B^{\top}\right)^{\top}$. After such matrix splitting, we can expand an accurate matrix multiplication by using floating-point matrix multiplications, which are performed fast by BLAS routines.

Algorithm 2.2 (Ozaki $[\mathbf{1 1}]$ ). For $A \in \mathbb{F}^{m \times n}$ and $B \in \mathbb{F}^{n \times p}$, this algorithm product $A B$ into unevaluated sum of floating-point matrices without rounding errors 
such that $A B=\sum_{i=1}^{h_{A} h_{B}} C_{i}, \quad C_{i} \in \mathbb{F}^{m \times n}$.

$$
\begin{aligned}
& \text { function } C_{h_{a} h_{B}}=\operatorname{EFT}_{-} \mathrm{mm}(A, B) ; \\
& {[m, n]=\operatorname{size}(A) ;[n, p]=\operatorname{size}(B) ;} \\
& D_{1: h_{A}}=\text { Ozaki_Split }(A) ; h_{A}:=\operatorname{length}(D) ; \\
& E_{1: h_{B}}=\text { Ozaki_Split }\left(B^{\top}\right) ; h_{B}:=\operatorname{length}(E) ; \\
& \text { for } i=1: h_{B}, \quad E_{i}=E_{i}^{\top} ; \\
& \text { for } i=1: h_{A} \\
& \qquad \begin{array}{c}
\text { for } j=1: h_{B} \\
C_{t}=\mathrm{f}\left(D_{i} \cdot E_{j}\right)
\end{array}
\end{aligned}
$$

After this transformation, we can guarantee a result with $k$-hold accuracy of the matrix product. Note that we need only these two cases $l=1$ and $l=k$ in (4). We use an accurate summation algorithm SumKK and SumK1 for $\sum_{i=1}^{h_{A} h_{B}} C_{i}$.

Let $x, y \in \mathbb{F}^{n}$. The following Knuth's algorithm transforms any pair of floatingpoint numbers $(a, b)$ into a new pair $(x, y)$ with

$$
x+y=a+b \quad \text { and } \quad x=\mathrm{f}(a+b) .
$$

ALGORITHM 2.3 (Knuth [4]). Error-free transformation for the sum of two floatingpoint numbers.

$$
\begin{aligned}
& \text { function }[x, y]=\text { Twosum }(a, b) \\
& \begin{array}{l}
x=\mathrm{f}(a+b) \\
z=\mathrm{fl}(x-a) \\
y=\mathrm{f}(a-(x-z)+(b-z))
\end{array}
\end{aligned}
$$


Let $p_{i}, q_{i} \in \mathbb{F}$ for $1 \leq i \leq n$. The vector $p_{1} \ldots, p_{n}$ is transformed into the vector $\pi, q_{2}, \ldots, q_{n}$, where the element $\pi$ is the ordinary floating point summation $\mathrm{fl}\left(\sum_{i=1}^{n} p_{i}\right)$ and the value of the sum does not change as $\sum_{i=1}^{n} p_{i}=\pi+\sum_{i=2}^{n} q_{i}$. This transformation is the following:

Algorithm 2.4 (Ogita et al. [8]). Error-free vector transformation for summation.

$$
\begin{aligned}
& \text { function } q=\operatorname{VecSum}(p) \\
& \qquad \begin{array}{l}
q_{n}=p_{n} \\
\text { for } i=n-1:-1: 1 \\
\qquad\left[q_{i}, q_{i+1}\right]=\operatorname{TwoSum}\left(p_{i}, p_{i+1}\right)
\end{array}
\end{aligned}
$$

We introduce an algorithm of the summation in $k$-fold precision and rounding into $k$ results. Assume $n \geq k$.

Algorithm 2.5. Summation as if computed in $k$-fold precision and rounded into $k$ results res $_{1: k}$

$$
\begin{aligned}
& \text { function } \text { res }_{1: k}=\operatorname{SumKK}(p, k) \\
& \text { for } i=1: k-1 \\
& p=\operatorname{VecSum}(p) \\
& \text { for } i=1: k-1 \\
& \operatorname{res}_{i}=p_{i} \\
& \operatorname{res}_{k}=\mathrm{fl}\left(\sum_{i=k}^{n} p_{i}\right)
\end{aligned}
$$


Algorithm 2.6 (Ogita [8]). Summation as if computed in $k$-fold precision and rounded into one result res.

$$
\begin{aligned}
& \text { function res }=\operatorname{SumK1}(p, k) \\
& \qquad \begin{array}{c}
p=\operatorname{Vec} \operatorname{Sum}(p) \\
\text { for } i=1: k-1 \\
\text { res }\left(\sum_{i=1}^{n} p_{i}\right)
\end{array}
\end{aligned}
$$

As mentioned before there is the fast error-free transformation of a product of two floating-point matrices $A, B$ into a sum of floating-point matrices. If this sum is calculated by SumKK and SumK1 $[\mathbf{8}, \mathbf{1 6}]$, we can have the result of the dot product satisfying (4).

\subsection{Accurate and robust inverse Cholesky factorization}

The following scheme compute the Cholseky factorization:

Algorithm 2.7 (Algorithm $10.2[\mathbf{3}]$ ). Give a symmetric positive definite $A=$ $\left(a_{i j}\right) \in \mathbb{R}^{n \times n}$. This algorithm computes the Cholesky factrization $A=R^{\top} R$.

for $j=1: n$

$$
\text { for } \begin{aligned}
i & =1: j-1 \\
r_{i j} & =\left(a_{i j}-\sum_{k=1}^{i-1} r_{k i} r_{k j}\right) / r_{i i}
\end{aligned}
$$

end

$$
r_{j j}=\left(a_{j j}-\sum_{k=1}^{j-1} r_{k j}^{2}\right)^{\frac{1}{2}}
$$


Let $A \in \mathbb{F}^{n \times n}$ be symmetric and positive definite. The Matlab-style notation

$$
R:=\operatorname{chol}(A)
$$

means a floating-point Cholesky factorization of $A$ using a standard numerical algorithm (e.g., xPOTRF in LAPACK) such that $A \approx R^{\top} R$ where $R \in \mathbb{F}^{n \times n}$ is an upper triangular matrix. We say "run to completion" if no imaginary root appears in the factorization process. It is well known $[\mathbf{3}]$ that the computed Cholesky factor $R$ always satisfies

$$
\frac{\left\|A-R^{\top} R\right\|}{\|A\|} \leq \varphi(\mathbf{u})
$$

if the floating-point Cholesky factorization runs to completion. From the residual norm $\left\|A-R^{\top} R\right\|$ or its relative norm, only limited information on the accuracy of the Cholesky factorization can be obtained. Suppose the exact Cholesky factorization of $A$ runs to completion such that $A=\hat{R}^{\top} \hat{R}$. Instead of (14), we use the following criterion:

$$
\left\|I-X^{\top} A X\right\|<\varepsilon_{t o l}
$$

where $X$ is an approximate inverse of $\hat{R}$ and $\varepsilon_{t o l}$ is a given tolerance, as the accuracy of the Cholesky factors.

As mentioned before, a floating-point Cholesky factorization of an ill-conditioned matrix $A$ may break down due to the accumulation of the rounding errors. To avoid the break-down, a diagonal shift can be applied to $A$ such as $\tilde{A}=f(A+\delta I)$ for some suitable $\delta>0$. It is advisable to obtain an optimal $\delta$ before executing $\operatorname{chol}(\tilde{A})$. Fortunately, it is possible. Rump [15] presented the following backward error bound of a floating-point Cholesky factorization based on the Demmel's result [1]: if $2(n+1) \mathbf{u}<$ 1 , then

$$
\Delta:=A-R^{T} R, \quad\|\Delta\| \leq c_{n}^{\prime} \mathbf{u} \cdot \operatorname{tr}(A), \quad c_{n}^{\prime}:=\frac{n+1}{1-2(n+1) \mathbf{u}} .
$$


Lemma 2.1 (cf. Theorem 2.3 in [17]). Let $A=A^{T} \in \mathbb{F}^{n \times n}$. If $\lambda_{n}(A) \geq c_{n}^{\prime} u \cdot \operatorname{tr}(A)$, then $\operatorname{chol}(A)$ runs to completion barring overflow and underflow.

In $[7,22]$, a diagonal shift is applied. In this thesis, we modified the diagonal shift of $A$ for the sake of completeness using Lemma 2.1. Assume $A$ is positive definite. Then $a_{i i}>0$ for all $i$ and $\lambda_{n}(A)>0$. From (2), it holds for a diagonal matrix $D=\left(d_{i j}\right) \in \mathbb{R}^{n \times n}$ that

$$
\tilde{A}=A+\delta I+D, \quad\left|d_{i i}\right| \leq \mathbf{u}\left(a_{i i}+\delta\right) .
$$

From Lemma 2.1, if $\lambda_{n}(\tilde{A}) \geq c_{n}^{\prime} \mathbf{u} \cdot \operatorname{tr}(\tilde{A})$, then $\operatorname{chol}(\tilde{A})$ never breaks down. Here

$$
\begin{aligned}
c_{n}^{\prime} \mathbf{u} \cdot \operatorname{tr}(\tilde{A}) & =c_{n}^{\prime} \mathbf{u} \cdot \operatorname{tr}(A+\delta I+D) \leq c_{n}^{\prime} \mathbf{u} \cdot \operatorname{tr}(A+\delta I+|D|) \\
& \leq c_{n}^{\prime} \mathbf{u}(1+\mathbf{u})(\operatorname{tr}(A)+n \delta) .
\end{aligned}
$$

On the other hand, by a Weyl's theorem (e.g. Corollary 4.9 in [21]) it holds

$$
\lambda_{n}(\tilde{A}) \geq \lambda_{n}(A+\delta I)-\|D\|>\delta-\max _{1 \leq i \leq n}\left|d_{i i}\right|
$$

and

$$
\max _{1 \leq i \leq n}\left|d_{i i}\right| \leq \max _{1 \leq i \leq n} \mathbf{u}\left(a_{i i}+\delta\right)=\mathbf{u} \max _{1 \leq i \leq n} a_{i i}+\delta \mathbf{u} \leq \mathbf{u} \cdot \operatorname{tr}(A)+\delta \mathbf{u}
$$

so that

$$
\lambda_{n}(\tilde{A}) \geq \delta-(\mathbf{u} \cdot \operatorname{tr}(A)+\delta \mathbf{u})=(1-\mathbf{u}) \delta-\mathbf{u} \cdot \operatorname{tr}(A)
$$

From (16) and (17), a sufficient condition such that $\operatorname{chol}(\tilde{A})$ runs to completion is

$$
(1-\mathbf{u}) \delta-\mathbf{u} \cdot \operatorname{tr}(A) \geq c_{n}^{\prime} \mathbf{u}(1+\mathbf{u})(\operatorname{tr}(A)+n \delta)
$$

and

$$
\delta \geq \frac{(1+\mathbf{u}) c_{n}^{\prime}+1}{1-\left((1+\mathbf{u}) n c_{n}^{\prime}+1\right) \mathbf{u}} \mathbf{u} \cdot \operatorname{tr}(A)
$$


If $(n+1)(n+3) \mathbf{u}<1$, then a little computation yields

$$
\frac{(1+\mathbf{u}) c_{n}^{\prime}+1}{1-\left((1+\mathbf{u}) n c_{n}^{\prime}+1\right) \mathbf{u}} \leq \frac{n+2}{1-(n+1)(n+3) \mathbf{u}}=: c_{n}
$$

Thus, if we determine $\delta$ satisfying $\delta \geq c_{n} \mathbf{u} \cdot \operatorname{tr}(A)$, then $\operatorname{chol}(\tilde{A})$ runs to completion. If $(n+1)(n+3) \mathbf{u}<1$, then we define a diagonal shift of $A \in \mathbb{F}^{n \times n}$ by

$$
\operatorname{shift}(A):=c_{n} \mathbf{u} \cdot \operatorname{tr}(A) \quad \text { with } \quad c_{n}=\frac{n+2}{1-(n+1)(n+3) \mathbf{u}} .
$$

Theorem 2.1. Let $A=A^{T} \in \mathbb{F}^{n \times n}$. Assume $(n+1)(n+3) \mathbf{u}<1$. Let $\operatorname{shift}(A)$ be defined as in (19). For any positive $\delta \in \mathbb{F}$ satisfying $\delta \geq \operatorname{shift}(A)$, put $\tilde{A}:=$ $f(A+\delta I)$. If $A$ is positive definite, then $\operatorname{chol}(\tilde{A})$ runs to completion barring overflow and underflow.

Let $\tilde{R}:=\operatorname{chol}(\tilde{A})$. Define

$$
\tilde{\Delta}:=\tilde{A}-\tilde{R^{\top}} \tilde{R}
$$

then it holds that $\|\tilde{\Delta}\| \leq \delta$. For $n \mathbf{u}<1$, we have $c_{n} \approx n$ and

$$
\|\tilde{\Delta}\| \leq c_{n} \mathbf{u} \cdot \operatorname{tr}(A) \leq c_{n} \mathbf{u} \cdot n\|A\| \approx n^{2} \mathbf{u}\|A\|
$$

The following algorithm calculates an upper triangular matrix $X_{1: m_{k}}^{(k)} \in \mathbb{F}_{\left[m_{k}\right]}^{n \times n}$ for some $k \in \mathbb{N}$ such that $\left\|X_{1: m_{k}}^{(k)} A X_{1: m_{k}}^{(k)}-I\right\|<\varepsilon_{t o l}$ for a symmetric matrix $A=\left(a_{i j}\right) \in$ $\mathbb{F}^{n \times n}$ with $a_{i i}>0$ for all $i$ and a specified tolerance $\varepsilon_{t o l}$ satisfying $n^{2} u \leq \varepsilon_{t o l} \leq 1$. 
Algorithm 2.8 (Ogita-Oishi [7]). Accurate inverse Cholesky factorization.

$$
\begin{aligned}
& \text { function }\left[X_{1: m_{k}}^{(k)}\right]=\text { acc_chol }\left(A, \varepsilon_{t o l}\right) \\
& k=0, \quad G^{(0)}:=A, \quad E^{(0)}:=O, \quad X_{1: 1}^{(0)}:=I \\
& \text { repeat } \\
& k=k+1 \\
& \text { Compute } S^{(k)} \in \mathbb{F}^{n \times n} \text { with } \\
& \left\{\begin{array}{l}
S_{i i}^{(k)}=f_{\triangle}\left(G_{i i}^{(k-1)}+\left\|E^{(k-1)}\right\|_{\infty}\right) \\
S_{i j}^{(k)}=G_{i j}^{(k-1)} \text { for } i \neq j .
\end{array} .\right. \\
& \delta_{k}:=\operatorname{shift}\left(S^{(k)}\right) \\
& \tilde{S}^{(k)}:=f l\left(S^{(k)}+\delta_{k} I\right) \\
& R^{(k)}:=\operatorname{chol}\left(\tilde{S}^{(k)}\right) \\
& T^{(k)}:=\operatorname{triinv}\left(R^{(k)}\right) \\
& m_{k}:=\left\lceil\frac{k}{2}\right\rceil+1 \\
& X_{1: m_{k}}^{(k)}:=\left\{X_{1: m_{k-1}}^{(k-1)} T^{(k)}\right\}_{m_{k}}^{m_{k}} \\
& \left\langle G^{(k)}, E^{(k)}\right\rangle:=\left\{\left(X_{1: m_{k}}^{(k)}\right)^{\top} A X_{1: m_{k}}^{(k)}\right\}_{k+1}^{1} \\
& \text { until } \quad f l_{\triangle}\left(\left\|G^{(k)}-I\right\|_{\infty}+\left\|E^{(k)}\right\|_{\infty}\right)<\varepsilon_{t o l}
\end{aligned}
$$

REMARK 2. Note that high precision computations of dot product are necessary only in (27) and (28). In [7], to determine how much precision is used for calculating $X^{(k-1)} T^{(k)}$ and $\left(X^{(k)}\right)^{\top} A X^{(k)}$, we needed to compute $\left\lceil-\log \left(\left\|X^{(k-1)}\right\|\|T\|\right) / \log \mathbf{u}\right\rceil+1$ and $\left\lceil-\log \left(\|A\|\left\|X^{(k)}\right\|^{2}\right) / \log \mathbf{u}\right\rceil+2$, respectively. In Chapter 3, we will make sure that the computational precision in (27) and (28) are appropriate. 
REMARK 3. In (21) and (22), we slightly shift the diagonal part of $G^{(k-1)}$ to ensure the positive definiteness of $\tilde{S}^{(k)}$ with taking care of the rounding errors. For (21), in practice, $S^{(k)}$ can be obtained by calculating $G_{i i}^{(k-1)}+\left\|E^{(k-1)}\right\|_{\infty}$ in rounding upwards.

Moreover, we introduce the following theorem for later use.

Theorem 2.2 (Theorem 1 in $[\mathbf{7}]$ ). Let $A$ be a symmetric $n \times n$ matrix. If Algorithm 2.8 successfully stops at (29) for any $\varepsilon_{\text {tol }} \leq 1$, then $A$ is positive definite. 
Chapter 3

\section{ConvergenCE ANALYSis OF AN}

\section{ALGORITHM FOR ACCURATE INVERSE \\ Cholesky FaCtorization}


This section is structured as follows. In Section 3.1, we analyze Algorithm 2.8 in detail and explain its convergency. In Section 3.2, we present some numerical results for confirming our analysis and comparing the algorithm with a standard Cholesky factorization by using long precision arithmetic $[\mathbf{2}, \mathbf{5}]$, in terms of measured computing time for verifying the positive definiteness of an input matrix.

\subsection{AnALYSis}

Following we analyze Algorithm 2.8 for input matrix $A \in \mathbb{F}^{n \times n}$ which is symmetric, positive definite and extremely ill-conditioned such that (1). The target of this section is to explain that the condition number of a preconditioned matrix $\left(X_{1: m_{k}}^{(k)}\right)^{\top} A X_{1: m_{k}}^{(k)}$ eventually converges to 1 after some iterations. As is the case with [13], we do not give a totally rigorous proof, so we frequent use the notation " $\approx$ " to mean that two quantities are the same order of magnitude. We also use the the notation "¿". For example, for $x, y \in \mathbb{R}, x \approx y$ means that $|x| \leq \varphi \cdot|y|$ for reasonably small constant $\varphi$. By the numerical results in [7], we observe the condition number of a preconditioned matrix is reduced by a factor around $n^{2} \mathbf{u}$ until convergence. Hence we expect

$$
\kappa\left(\left(X_{1: m_{k}}^{(k)}\right)^{\top} A X_{1: m_{k}}^{(k)}\right) \approx 1+\prod_{i=1}^{k} \alpha_{i} \cdot \kappa(A), \quad \alpha_{i}:=\frac{\delta_{i}}{\left\|\left(X_{1: m_{i-1}}^{(i-1)}\right)^{\top} A X_{1: m_{i-1}}^{(i-1)}\right\|}
$$

Assume $\left\|E^{(k-1)}\right\| \approx \mathbf{u}\left\|G^{(k-1)}\right\|$. Then

$$
\delta_{k}=c_{n} \mathbf{u} \cdot \operatorname{tr}\left(S^{(k)}\right) \approx n \mathbf{u} \cdot \operatorname{tr}\left(G^{(k-1)}\right)
$$

For this, it holds that $n \mathbf{u} \cdot \operatorname{tr}\left(G^{(k-1)}\right) \geq n \mathbf{u}\left\|G^{(k-1)}\right\|$ and $n \mathbf{u} \cdot \operatorname{tr}\left(G^{(k-1)}\right) \leq n^{2} \mathbf{u}\left\|G^{(k-1)}\right\|$. Therefore, $\delta_{k}$ can be understood as

$$
n \mathbf{u}\left\|G^{(k-1)}\right\| \lesssim \delta_{k} \lesssim n^{2} \mathbf{u}\left\|G^{(k-1)}\right\| .
$$


Define $\alpha_{k}:=\delta_{k} /\left\|\left(X_{1: m_{k-1}}^{(k-1)}\right)^{\top} A X_{1: m_{k-1}}^{(k-1)}\right\|$. Then $\left\|\left(X_{1: m_{k-1}}^{(k-1)}\right)^{\top} A X_{1: m_{k-1}}^{(k-1)}\right\| \approx\left\|G^{(k-1)}\right\|$ and

$$
n \mathbf{u} \lesssim \alpha_{k} \lesssim n^{2} \mathbf{u}
$$

Consequently, (30) can be understood as

$$
\kappa\left(X_{1: m_{k}}^{(k) \top} A X_{1: m_{k}}^{(k)}\right) \approx 1+\left(n^{2} \mathbf{u}\right)^{k} \cdot \kappa(A)
$$

3.1.1. Preparation for analysis. In the same way as $[7]$, the conditioned number of a computed Cholesky factor of ill-conditioned symmetric positive matrix is limited to not much more than around $\mathbf{u}^{-\frac{1}{2}}$.

Observation 3.1. Let $A=A^{\top} \in \mathbb{F}^{n \times n}$ be positive definite. For $\delta:=\operatorname{shift}(A)$, put $\tilde{A}:=f l(A+\delta I)$. Let $R:=\operatorname{chol}(\tilde{A})$. Define $\alpha:=\delta /\|A\|$. Then

$$
\kappa(R) \approx \sqrt{\min \left\{\kappa(A), \alpha^{-1}\right\}}
$$

Argument If $\kappa(A)>u^{-1}$, then

$$
\kappa(\tilde{A}) \approx \kappa(A+\delta I) \approx \frac{\|A\|}{\delta} \approx \alpha^{-1}
$$

and $\kappa(R) \approx \alpha^{-\frac{1}{2}}$. Otherwise, $\kappa(\tilde{A}) \approx \kappa(A)$. Therefore, $\tilde{A}$ is not too ill-conditioned in any case and

$$
\kappa(R) \approx \kappa(\tilde{A})^{\frac{1}{2}} \approx \kappa(A)^{\frac{1}{2}}
$$

Thus, we have

$$
\kappa(R) \approx \sqrt{\min \left\{\kappa(A), \alpha^{-1}\right\}}
$$

We introduce the following lemmas for bounds on an inversion of a matrix. 
Lemma 3.1 (Lemma 14.1 in Higham [3]). Let $R \in \mathbb{F}^{n \times n}$ be a triangular matrix. Let $X:=\operatorname{triinv}(R)$. Then

$$
|I-R X| \leq \gamma_{n}|R||X| \quad \text { where } \quad \gamma_{n}=\frac{n \mathbf{u}}{1-n \mathbf{u}} .
$$

Lemma 3.2. Let $R$ and $X$ be defined as in Lemma Table 3.1. Then

$$
\left\|I-X^{\top} R^{\top} R X\right\| \leq 2 \beta+\beta^{2}
$$

where $\beta:=\gamma_{n}\||R\|X \mid\|$.

Proof. From Lemma 3.1, we have

$$
\|I-R X\| \leq \beta
$$

and

$$
\begin{aligned}
\left\|I-X^{\top} R^{\top} R X\right\| & =\left\|I-\left(I-\left(I-X^{\top} R^{\top}\right)\right)(I-(I-R X))\right\| \\
& \leq 2\|I-R X\|+\|I-R X\|^{2} \\
& \leq 2 \beta+\beta^{2} .
\end{aligned}
$$

If $\beta \leq 1$ satisfies, then $\beta^{2} \leq 1$ and

$$
\left\|I-X^{\top} R^{\top} R X\right\| \approx n u\|R\|\|X\| .
$$

In the same way as Assumption 3.3 in [6], we present the following observation on the relationship between $R$ and $T$.

Observation 3.2. Let $A, R$ and $\delta$ be defined as in Observation 3.1. Let $T:=$ $\operatorname{triinv}(R)$. Then

$$
\|R T\| \approx 1 \quad \text { and } \quad\left\|(R T)^{-1}\right\| \approx 1 .
$$


Argument Define $\alpha:=\delta /\|A\|$. From (32), we have $n \mathbf{u} \lesssim \alpha \lesssim n^{2} \mathbf{u}$. Observation 3.1 implies $\kappa(R) \approx \sqrt{\min \left(\kappa(A), \alpha^{-1}\right)}$. Therefore, $\|R\| T \mid \| \leq \kappa(R) \approx \alpha^{-\frac{1}{2}}$. From Lemma Table 3.1, we can expect in any case (35) holds.

In the same way as Observation 3.6 in [16], we present the following observation for the estimations of a norm of $T$ and $T^{-1}$, respectively.

Observation 3.3. Let $A, R, T$ and $\delta$ be defined as in Observation 3.2. Define $\mu:=\min \left(\kappa(A), \alpha^{-1}\right)$ with $\alpha:=\delta /\|A\|$. Then

$$
\|T\| \approx \frac{\mu^{\frac{1}{2}}}{\|R\|} \quad \text { and } \quad\left\|T^{-1}\right\| \approx\|R\| .
$$

Argument By Observation 3.1, $\kappa(R)$ can be expected to be not much larger than $\alpha^{-\frac{1}{2}}$. Thus, we can expect in any case $\|T\| \approx \mu^{\frac{1}{2}} /\|R\|$. Since $T^{-1}=(R T)^{-1} R$, we have

$$
\frac{\|R\|}{\|R T\|} \leq\left\|T^{-1}\right\| \leq\left\|(R T)^{-1}\right\|\|R\|
$$

From Observation 3.2, $\|R T\| \approx 1$ and $\left\|(R T)^{-1}\right\| \approx 1$. Hence, $\left\|T^{-1}\right\| \approx\|R\|$.

Next, we introduce the Rump's lemma in [16] for an estimation of a norm of a matrix.

Lemma 3.3 (Lemma 3.7 in [16]). Let a matrix $A \in \mathbb{R}^{n \times n}$ and a vector $x \in \mathbb{R}^{n}$ be given which are not correlated. Suppose $n \geq 4$. Then

$$
\|A\| \geq E\left(\frac{\|A x\|}{\|x\|}\right) \geq \frac{0.61}{\sqrt{n-1}}\|A\|,
$$

where $E(\cdot)$ denotes an expectation value.

This means $\|A\|\|x\| \approx\|A x\|$ in general.

3.1.2. Convergence. To explain (30), we first consider the case of $k=1$ in Algorithm 2.8. 
AnAlysis 1. We utilize (22)-(28) at the first iteration in Algorithm 2.8. For readability, $R^{(1)}, T^{(1)}$ and $X_{1: m_{1}}^{(1)}$ are abbreviated to $R_{1}, T_{1}$ and $X_{1}$, respectively. Define

$$
\mu_{1}:=\min \left(\kappa(A), \alpha_{1}^{-1}\right) \quad \text { with } \quad \alpha_{1}:=\frac{\delta_{1}}{\|A\|}
$$

If $A \in \mathbb{F}^{n \times n}$ is positive definite, $(n+1)(n+3) \mathbf{u}<1$ and we can use high precision computations for dot product which satisfies (4), we analyze to show

$$
\begin{aligned}
& \left\|X_{1}\right\| \approx\left(\frac{\mu_{1}}{\|A\|}\right)^{\frac{1}{2}}, \\
& \left\|X_{1}^{-1}\right\| \approx\|A\|^{\frac{1}{2}}, \\
& \left\|X_{1}^{\top} A X_{1}\right\| \approx 1 \text { and } \\
& \kappa\left(X_{1}^{\top} A X_{1}\right) \approx 1+\alpha_{1} \kappa(A) .
\end{aligned}
$$

REMARK 4. Note that for ill-conditioned A, i.e. $\kappa(A)>\alpha_{1}^{-1}$, we have $\mu_{1}=\alpha_{1}^{-1}$, and the estimations (36) and (39) can be understood as

$$
\left\|X_{1}\right\| \approx\left(\alpha_{1}\|A\|\right)^{-\frac{1}{2}}
$$

REMARK 5. From (32), it holds that

$$
n \mathbf{u} \lesssim \alpha_{1} \lesssim n^{2} \mathbf{u}
$$

\section{Argument Define}

$$
\Delta_{1}:=A-R_{1}^{\top} R_{1}
$$

From (15) and (20), it holds

$$
\left\|\Delta_{1}\right\| \leq\|A-\tilde{A}\|+\left\|\tilde{A}-R_{1}^{\top} R_{1}\right\| \approx \alpha_{1}\|A\|+\alpha_{1}\|A\| \approx \alpha_{1}\|A\|
$$

and

$$
\left\|R_{1}\right\| \approx\|A\|^{\frac{1}{2}}
$$


By Observation 3.3 and (42), we have

$$
\left\|X_{1}\right\|=\left\|T_{1}\right\| \approx \frac{\mu_{1}^{\frac{1}{2}}}{\left\|R_{1}\right\|} \approx\left(\frac{\mu_{1}}{\|A\|}\right)^{\frac{1}{2}}
$$

and

$$
\left\|X_{1}^{-1}\right\| \approx\|A\|^{\frac{1}{2}}
$$

which explain (36) and (37), respectively.

Next we estimate $\left\|I-X_{1}^{\top} A X_{1}\right\|$ to show (38). It holds

$$
\begin{aligned}
\left\|I-X_{1}^{\top} A X_{1}\right\| & =\left\|I-X_{1}^{\top}\left(A-R_{1}^{\top} R_{1}+R_{1}^{\top} R_{1}\right) X_{1}\right\| \\
& \leq\left\|I-X_{1}^{\top} R_{1}^{\top} R_{1}\right\|+\left\|X_{1}^{\top}\left(A-R_{1}^{\top} R_{1}\right) X_{1}\right\| \\
& \leq\left\|I-X_{1}^{\top} R_{1}^{\top} R_{1} X_{1}\right\|+\left\|X_{1}^{\top} \Delta_{1} X_{1}\right\| .
\end{aligned}
$$

From (36) and (42), we have

$$
\left\|R_{1}\right\|\left\|X_{1}\right\| \approx \mu_{1}^{\frac{1}{2}}
$$

If $A$ is ill-conditioned, i.e., $\kappa(A) \geq \alpha_{1}^{-1}$, then

$$
\gamma_{n}\left\|\left|R_{1}\left\|X_{1} \mid\right\| \approx n \mathbf{u} \cdot \alpha_{1}^{-\frac{1}{2}} \lesssim(n \mathbf{u})^{\frac{1}{2}}<1\right.\right.
$$

under the assumption $(n+1)(n+3) \mathbf{u}<1$ and from (40). Otherwise,

$$
\gamma_{n}\left\|\left|R_{1}\left\|X_{1} \mid\right\| \approx n \mathbf{u} \cdot \kappa(A)^{\frac{1}{2}}<1\right.\right.
$$

Then, applying (34) yields

$$
\left\|I-X_{1}^{\top} R_{1}^{\top} R_{1} X_{1}\right\| \approx n \mathbf{u}\left\|R_{1}\right\|\left\|X_{1}\right\| \approx n \mathbf{u} \mu_{1}^{\frac{1}{2}}
$$


Moreover, using (41), (42) and (44), we have

$$
\begin{aligned}
\left\|X_{1}^{\top} \Delta_{1} X_{1}\right\| & \leq\left\|X_{1}^{\top}\right\|\left\|\Delta_{1}\right\|\left\|X_{1}\right\| \approx \alpha_{1}\|A\|\left\|X_{1}\right\|^{2} \approx \alpha_{1}\left(\left\|R_{1}\right\|\left\|X_{1}\right\|\right)^{2} \\
& \approx \alpha_{1} \mu_{1} .
\end{aligned}
$$

Inserting (47) and (48) into (43) implies

$$
\left\|I-X_{1}^{\top} A X_{1}\right\| \approx n \mathbf{u} \mu_{1}^{\frac{1}{2}}+\alpha_{1} \mu_{1} \approx \alpha_{1} \mu_{1} .
$$

which explain (38). If $A$ is ill-conditioned, i.e., $\kappa(A) \geq \alpha_{1}^{-1}$, then $\alpha_{1} \mu_{1}=\alpha_{1} \alpha_{1}^{-1} \approx 1$. Otherwise, $\alpha_{1} \mu_{1}=\alpha_{1} \kappa(A)<1$. Then, we have

$$
\left\|X_{1}^{\top} A X_{1}\right\| \approx 1
$$

Finally, we estimate $\left\|\left(X_{1}^{\top} A X_{1}\right)^{-1}\right\|$ to show (39). It holds that

$$
\begin{aligned}
\left\|\left(X_{1}^{\top} A X_{1}\right)^{-1}\right\| & =\left\|\left(R_{1} X_{1}\right)^{-1}\left(R_{1} A^{-1} R_{1}^{\top}\right)\left(\left(R_{1} X_{1}\right)^{\top}\right)^{-1}\right\| \\
& \leq\left\|\left(R_{1} X_{1}\right)^{-1}\right\|^{2}\left\|R_{1} A^{-1} R_{1}^{\top}\right\| .
\end{aligned}
$$

Here, applying Observation 3.2 yields

$$
\left\|\left(R_{1} X_{1}\right)^{-1}\right\| \approx 1 .
$$

Let $\hat{R}_{1}$ denote the exact Cholesky factor of $A$ such that $A=\hat{R}_{1}^{\top} \hat{R}_{1}$. Then

$$
\begin{aligned}
\left\|R_{1} A^{-1} R_{1}^{\top}\right\| & =\left\|\left(R_{1} \hat{R}_{1}^{-1}\right)\left(\left(\hat{R}_{1}^{\top}\right)^{-1} R_{1}^{\top}\right)\right\|=\left\|\left(R_{1} \hat{R}_{1}^{-1}\right)^{\top}\left(R_{1} \hat{R}_{1}^{-1}\right)\right\| \\
& =\left\|\left(\hat{R}_{1}^{\top}\right)^{-1} R_{1}^{\top} R_{1} \hat{R}_{1}^{-1}\right\|=\left\|\left(\hat{R}_{1}^{\top}\right)^{-1}\left(A+\Delta_{1}\right) \hat{R}_{1}^{-1}\right\| \\
& \leq 1+\left\|\left(\hat{R}_{1}^{\top}\right)^{-1} \Delta_{1} \hat{R}_{1}^{-1}\right\| \leq 1+\left\|\Delta_{1}\right\|\left\|\hat{R}_{1}^{-1}\right\|^{2} \\
& =1+\left\|\Delta_{1}\right\|\left\|A^{-1}\right\| .
\end{aligned}
$$


From (41) and (52), we have

$$
\left\|R_{1} A^{-1} R_{1}^{\top}\right\| \approx 1+\alpha_{1} \kappa(A) .
$$

Inserting (51) and (53) into (50) implies

$$
\left\|\left(X_{1}^{\top} A X_{1}\right)^{-1}\right\| \approx 1+\alpha_{1} \kappa(A)
$$

This and (49) explain (39).

Next, suppose we are at the $(k+1)$-st iteration in Algorithm 2.8.

AnALYsis 2. We utilize (21)-(28) at the $(k+1)$-st iteration in Algorithm 2.8 and the estimates in Analysis 1. For readability, $G^{(k)}, X_{1: m_{k}}^{(k)}, S^{(k+1)}, \tilde{S}^{(k+1)}, R^{(k+1)}$ and $X_{1: m_{k+1}}^{(k+1)}$, are abbreviated to $G, X, S, \tilde{S}, R$ and $X^{\prime}$, respectively. Define

$$
\mu:=\min \left(\kappa\left(X^{\top} A X\right), \alpha_{k+1}^{-1}\right) \quad \text { with } \quad \alpha_{k+1}:=\frac{\delta_{k+1}}{\left\|X^{\top} A X\right\|}
$$

Assume that $A \in \mathbb{F}^{n \times n}$ is positive definite, $(n+1)(n+3) \mathbf{u}<1$ and we can use high precision computations for dot product which satisfies (4). Moreover, assume that

$$
\begin{aligned}
& \|X\| \approx\left(\prod_{i=1}^{k} \alpha_{i} \cdot\|A\|\right)^{-\frac{1}{2}}, \\
& \left\|X^{-1}\right\| \approx\|A\|^{\frac{1}{2}}, \\
& \left\|X^{\top} A X\right\| \approx 1 \text { and } \\
& \kappa\left(X^{\top} A X\right) \approx 1+\prod_{i=1}^{k} \alpha_{i} \cdot \kappa(A) .
\end{aligned}
$$


Then we analyze

$$
\begin{aligned}
& \left\|X^{\prime}\right\| \approx \mu^{\frac{1}{2}}\|X\|, \\
& \left\|X^{\prime-1}\right\| \approx\|A\|^{\frac{1}{2}}, \\
& \left\|X^{\prime \top} A X^{\prime}\right\| \approx 1 \text { and } \\
& \kappa\left(X^{\prime \top} A X^{\prime}\right) \approx 1+\alpha_{k+1} \cdot \kappa\left(X^{\top} A X\right) .
\end{aligned}
$$

REMARK 6. Note that for ill-conditioned $X^{\top} A X$, i.e. $\kappa\left(X^{\top} A X\right) \geq \alpha_{k+1}^{-1}$, we have $\mu=\alpha_{k+1}^{-1}$, and the estimations (59) and (62) can be understood as

$$
\left\|X^{\prime}\right\| \approx\left(\prod_{i=1}^{k+1} \alpha_{i} \cdot\|A\|\right)^{-\frac{1}{2}}
$$

and

$$
\kappa\left(X^{\prime \top} A X^{\prime}\right) \approx 1+\prod_{i=1}^{k+1} \alpha_{i} \kappa(A) \lesssim 1+\left(n^{2} \mathbf{u}\right)^{k+1} \kappa(A)
$$

REMARK 7. From (32), we have

$$
n \mathbf{u} \lesssim \alpha_{k+1} \lesssim n^{2} \mathbf{u}
$$

Argument Define

$$
\begin{aligned}
\Delta_{2} & :=X^{\top} A X-R^{\top} R, \\
\Delta_{3} & :=X^{\prime}-X T .
\end{aligned}
$$

From (21), (98), (23) and (28) in Algorithm 2.8, we have

$$
\begin{aligned}
& \left\|X^{\top} A X-G\right\| \leq\|E\|, \\
& \|G-S\|=\|E\|, \\
& \|S-\tilde{S}\| \approx \delta_{k+1}=\alpha_{k+1}\left\|X^{\top} A X\right\| .
\end{aligned}
$$


The computation of $X^{\top} A X$ by $\left\{X^{\top} A X\right\}_{k+1}^{1}$, (4) , (55), (57) and (63) imply

$$
\begin{aligned}
\|E\| & \leq \varphi(\mathbf{u})\left\|X^{\top} A X\right\|+\varphi\left(\mathbf{u}^{k+1}\right)\left\|X^{\top}\right\|\|A\|\|X\| \\
& \lesssim \mathbf{u} \cdot 1+\mathbf{u}^{\mathbf{k}+\mathbf{1}} \cdot\left((n \mathbf{u})^{\mathbf{k}}\right)^{-\mathbf{1}} \approx \mathbf{u} .
\end{aligned}
$$

From (57), (63), (66)-(69), it holds

$$
\begin{aligned}
\left\|X^{\top} A X-\tilde{S}\right\| & \leq\left\|X^{\top} A X-G\right\|+\|G-S\|+\|S-\tilde{S}\| \\
& \leq\|E\|+\|E\|+\alpha_{k+1}\left\|X^{\top} A X\right\| \\
& \approx \mathbf{u}+\mathbf{u}+\alpha_{k+1} \approx \alpha_{k+1} .
\end{aligned}
$$

This and (57) yield

$$
\|\tilde{S}\| \approx 1+\alpha_{k+1} \approx 1
$$

From (20) and (71), it holds

$$
\left\|\tilde{S}-R^{\top} R\right\| \approx \alpha_{k+1}\|\tilde{S}\| \approx \alpha_{k+1}
$$

On (64), from (70) and (72), we have

$$
\left\|\Delta_{2}\right\| \leq\left\|X^{\top} A X-\tilde{S}\right\|+\left\|\tilde{S}-R^{\top} R\right\| \approx \alpha_{k+1}+\alpha_{k+1} \approx \alpha_{k+1},
$$

and

$$
\|R\| \approx\|\tilde{S}\|^{\frac{1}{2}} \approx 1
$$

Hence Observation 3.3 and (74) yield

$$
\|T\| \approx \frac{\mu^{\frac{1}{2}}}{\|R\|} \approx \mu^{\frac{1}{2}}, \quad\left\|T^{-1}\right\| \approx\|R\| \approx 1 .
$$


On (65), the computation of $X T$ by $\{X T\}_{m_{k+1}}^{m_{k+1}}$ with $m_{k+1}=\left\lceil\frac{k+1}{2}\right\rceil+1$ gives

$$
\begin{aligned}
\left\|\Delta_{3}\right\| & \leq \varphi\left(\mathbf{u}^{\left\lceil\frac{k+1}{2}\right\rceil+1}\right)\|X T\|+\varphi\left(\mathbf{u}^{\left\lceil\frac{k+1}{2}\right\rceil+1}\right)\|X\|\|T\| \\
& \approx \mathbf{u}^{\left\lceil\frac{k+1}{2}\right\rceil+1}\|X\|\|T\| .
\end{aligned}
$$

under the assumption (4). Combining (55), (63), (75) and (76) yields

$$
\left\|\Delta_{3}\right\| \approx \mathbf{u}^{\left\lceil\frac{k+1}{2}\right\rceil+1}\left(\prod_{i=1}^{k} \alpha_{i} \cdot \mu^{-1}\|A\|\right)^{-\frac{1}{2}} \lesssim \mathbf{u}^{\left\lceil\frac{k+1}{2}\right\rceil+1}(n \mathbf{u})^{-\frac{k}{2}} \mu^{\frac{1}{2}}\|A\|^{-\frac{1}{2}}
$$

In the similar way to (45) and (46), we have

$$
\left\|\Delta_{3}\right\| \lesssim \mathbf{u}\|A\|^{-\frac{1}{2}}
$$

Now we estimate $\left\|X^{\prime}\right\|$ in (59). Here we consider the relation between $X^{\prime}$ and $X T$. Define $F:=\Delta_{3} T^{-1} X^{-1}$. Then

$$
X^{\prime}=(I+F) X T \text {. }
$$

Combining (56), (75) and (78) yields

$$
\|F\| \leq\left\|\Delta_{3}\right\|\left\|T^{-1}\right\|\left\|X^{-1}\right\| \approx \mathbf{u}
$$

and

$$
\|I+F\| \approx 1 \text { and }\left\|(I+F)^{-1}\right\| \approx 1
$$

From (79), it follows

$$
\frac{\|X T\|}{\left\|(I+F)^{-1}\right\|} \leq\left\|X^{\prime}\right\| \leq\|I+F\|\|X T\|
$$

Therefore, by Lemma 3.3, (75) and (82), it follows

$$
\left\|X^{\prime}\right\| \approx\|X T\| \approx\|X\|\|T\| \approx \mu^{\frac{1}{2}}\|X\|
$$


which explains (59).

Next we estimate $\left\|X^{\prime-1}\right\|$ in (60). In a similar way to (82), we have $\left\|X^{\prime-1}\right\| \approx$ $\left\|(X T)^{-1}\right\|$. Hence, from Lemma 3.3, (56) and (75), it follows

$$
\left\|X^{\prime-1}\right\| \approx\left\|(X T)^{-1}\right\| \approx\left\|T^{-1}\right\|\left\|X^{-1}\right\| \approx\|A\|^{\frac{1}{2}},
$$

which explains (60).

Next we estimate $\left\|I-X^{\prime \top} A X^{\prime}\right\|$ to show (61). Then

$$
\begin{aligned}
\left\|I-X^{\prime \top} A X^{\prime}\right\| & =\left\|I-\left(X T+\Delta_{3}\right)^{\top} A\left(X T+\Delta_{3}\right)\right\| \\
& \leq\left\|I-T^{\top} X^{\top} A X T\right\|+2\left\|T^{\top} X^{\top} A \Delta_{3}\right\|+\left\|\Delta_{3}^{\top} A \Delta_{3}\right\| .
\end{aligned}
$$

Here

$$
\begin{aligned}
\left\|I-T^{\top} X^{\top} A X T\right\| & =\left\|I-T^{\top}\left(R^{\top} R+\Delta_{2}\right) T\right\| \\
& \leq\left\|I-T^{\top} R^{\top} R T\right\|+\left\|T^{\top} \Delta_{2} T\right\| .
\end{aligned}
$$

In a similar way to (47), applying (34) yields

$$
\left\|I-T^{\top} R^{\top} R T\right\| \approx n \mathbf{u}\|T\|\|R\| \approx n \mathbf{u} \mu^{\frac{1}{2}} .
$$

Moreover, by (73) and (75), we have

$$
\left\|T^{\top} \Delta_{2} T\right\| \leq\left\|\Delta_{2}\right\|\|T\|^{2} \approx \alpha_{k+1} \mu .
$$

Inserting (85) and (86) into (84) implies

$$
\left\|I-T^{\top} X^{\top} A X T\right\| \approx n \mathbf{u} \mu^{\frac{1}{2}}+\alpha_{k+1} \mu \approx \alpha_{k+1} \mu .
$$


We estimate $\left\|T^{\top} X^{\top} A \Delta_{3}\right\|$ and $\left\|\Delta_{3}^{\top} A \Delta_{3}\right\|$ in (83). Using (57), (60), (63), (75) and (78), we have

$$
\begin{aligned}
\left\|T^{\top} X^{\top} A \Delta_{3}\right\| & =\left\|T^{\top}\left(X^{\top} A X\right) X^{-1} \Delta_{3}\right\| \leq\|T\|\left\|X^{\top} A X\right\|\left\|X^{-1}\right\|\left\|\Delta_{3}\right\| \\
& \lesssim \mathbf{u}^{\left\lceil\frac{k+1}{2}\right\rceil+1}(n \mathbf{u})^{-\frac{k}{2}} \mu^{\frac{1}{2}}
\end{aligned}
$$

In a similar way to (45) and (46), we have

$$
\left\|T^{\top} X^{\top} A \Delta_{3}\right\| \lesssim \mathbf{u}^{\left\lceil\frac{k+1}{2}\right\rceil+1}(n \mathbf{u})^{-\frac{k}{2}}(n \mathbf{u})^{-\frac{1}{2}} \approx \mathbf{u}
$$

Moreover, by (78), it holds

$$
\left\|\Delta_{3}^{\top} A \Delta_{3}\right\| \leq\left\|\Delta_{3}\right\|^{2}\|A\| \lesssim \mathbf{u}^{2}\|A\|^{-1}\|A\|=\mathbf{u}^{2}
$$

Inserting (87)-(89) into (83) implies

$$
\left\|I-X^{\prime \top} A X^{\prime}\right\| \leq \alpha_{k+1} \mu+\mathbf{u}+\mathbf{u}^{2} \approx \alpha_{k+1} \mu
$$

which explains (61).

To see (62), suppose first that $X^{\top} A X$ is not so ill-conditioned, i.e. $\kappa\left(X^{\top} A X\right) \leq$ $\alpha_{k+1}^{-1}$. Then the definition (54) implies $\mu=\kappa\left(X^{\top} A X\right)$. Thus we have

$$
\kappa\left(X^{\prime \top} A X^{\prime}\right) \approx 1 \approx \mu^{-1} \kappa\left(X^{\top} A X\right)
$$

Second, suppose $X^{\top} A X$ is extremely ill-conditioned, i.e. $\kappa\left(X^{\top} A X\right)>\alpha_{k+1}^{-1}$. Then the definition (54) implies $\mu=\alpha_{k+1}^{-1}$. From (79), it holds that

$$
\begin{aligned}
\left\|\left(X^{\prime \top} A X^{\prime}\right)^{-1}\right\| & =\left\|\left((X T(I+F))^{\top} A X T(I+F)\right)^{-1}\right\| \\
& \leq\left\|\left(T^{\top} X^{\top} A X T\right)^{-1}\right\|\left\|(I+F)^{-1}\right\|^{2} .
\end{aligned}
$$


On (91), it holds that

$$
\begin{aligned}
\left\|\left(T^{\top} X^{\top} A X T\right)^{-1}\right\| & =\left\|(R T)^{-1} R\left(X^{\top} A X\right)^{-1} R^{\top}\left((R T)^{\top}\right)^{-1}\right\| \\
& \leq\left\|(R T)^{-1}\right\|^{2}\left\|R\left(X^{\top} A X\right)^{-1} R^{\top}\right\| .
\end{aligned}
$$

Here, applying Observation 3.2 yields

$$
\left\|(R T)^{-1}\right\| \approx 1
$$

Let $\hat{R}$ denote the exact Cholesky factor of $X^{\top} A X$ such that $X^{\top} A X=\hat{R}^{\top} \hat{R}$. Then

$$
\begin{aligned}
\left\|R\left(X^{\top} A X\right)^{-1} R^{\top}\right\| & =\left\|R \hat{R}^{-1}\left(\hat{R}^{\top}\right)^{-1} R^{\top}\right\|=\left\|\left(R \hat{R}^{-1}\right)^{\top}\left(R \hat{R}^{-1}\right)\right\| \\
& =\left\|\left(\hat{R}^{\top}\right)^{-1} R^{\top} R \hat{R}^{-1}\right\|=\left\|\left(\hat{R}^{\top}\right)^{-1}\left(X^{\top} A X+\Delta_{2}\right) \hat{R}^{-1}\right\| \\
& \leq 1+\left\|\left(\hat{R}^{\top}\right)^{-1} \Delta_{2} \hat{R}^{-1}\right\| .
\end{aligned}
$$

Here, from (73) we have

$$
\left\|\left(\hat{R}^{\top}\right)^{-1} \Delta_{2} \hat{R}^{-1}\right\| \leq\left\|\Delta_{2}\right\|\left\|\left(X^{\top} A X\right)^{-1}\right\| \approx \alpha_{k+1}\left\|\left(X^{\top} A X\right)^{-1}\right\|
$$

and

$$
\left\|R\left(X^{\top} A X\right)^{-1} R^{\top}\right\| \approx 1+\alpha_{k+1}\left\|\left(X^{\top} A X\right)^{-1}\right\| .
$$

The assumption $\kappa\left(X^{\top} A X\right)>\alpha_{k+1}^{-1}$ with $\left\|X^{\top} A X\right\| \approx 1$ implies

$$
\left\|R\left(X^{\top} A X\right)^{-1} R^{\top}\right\| \approx 1+\alpha_{k+1}\left\|\left(X^{\top} A X\right)^{-1}\right\| .
$$

Inserting (93) and (94) into (92) yields

$$
\left\|\left(T^{\top} X^{\top} A X T\right)^{-1}\right\| \approx 1+\alpha_{k+1}\left\|\left(X^{\top} A X\right)^{-1}\right\| .
$$

Combining (81), (91) and (95), it follows that

$$
\left\|\left(X^{\prime \top} A X^{\prime}\right)^{-1}\right\| \approx 1 \cdot\left(1+\alpha_{k+1}\left\|\left(X^{\top} A X\right)^{-1}\right\|\right) \approx 1+\alpha_{k+1}\left\|\left(X^{\top} A X\right)^{-1}\right\| .
$$


From this, (57), (58) and (61), we have

$$
\kappa\left(X^{\prime \top} A X^{\prime}\right)=\left\|X^{\prime \top} A X^{\prime}\right\| \cdot\left\|\left(X^{\prime \top} A X^{\prime}\right)^{-1}\right\| \approx 1+\alpha_{k+1} \kappa\left(X^{\top} A X\right)
$$

which explains (62).

3.1.3. Computational complexity. The computational complexity in terms of time and space complexity of Algorithm 2.8 strongly depends on that of accurate matrix multiplication. We assume that an algorithm for accurate dot product based on the algorithms in $[\mathbf{8}, \mathbf{1 1}, \mathbf{1 6}]$ is used. For example, $A$ and $X_{1: m_{k}}^{(k)}$ are split into $\sum_{i=1}^{h_{A}} D_{i}$ and $\sum_{i=1}^{h_{X}} E_{i}$ without rounding errors, respectively. After such matrix splitting, we have $A X_{1: m_{k}}^{(k)}=\sum_{i=1}^{h_{A} h_{X}} C_{i}, C_{i} \in \mathbb{F}^{n \times n}$ which requires $\mathcal{O}\left(h_{A} h_{X} n^{3}\right)$ flops. In practice, we can observe $h_{A} h_{X} \approx k^{2}$ at the $k$-th iteration. Therefore, it requires $\mathcal{O}\left(k^{2} n^{3}\right)$ flops to compute the matrix multiplication $\left\{X_{1: m_{k-1}}^{(k-1)} T^{(k)}\right\}_{m_{k}}^{m_{k}}$ and $\left\{X_{1: m_{k}}^{(k) \top} A X_{1: m_{k}}^{(k)}\right\}_{k+1}^{1}$ and $\mathcal{O}\left(n^{3}\right)$ flops to compute the rest of the algorithm at the $k$-th iteration. If the algorithm stops at $k=m$, then the total time complexity of Algorithm 2.8 becomes $\mathcal{O}\left(m^{3} n^{3}\right)$ flops since $\sum_{k=1}^{m} k^{2} n^{3}=\mathcal{O}\left(m^{3} n^{3}\right)$. By our analysis, we can see that the number of iterations $m$ in Algorithm 2.8 as

$$
m_{1}=\left\lceil\frac{\log \varepsilon_{t o l}-\log \kappa(A)}{\log \left(n^{2} \mathbf{u}\right)}\right\rceil
$$

suffices to achieve (29).

\subsection{NumeriCAL EXPERIMENTS}

In this section we present some numerical results. All computations are done on Matlab 2011b and IEEE standard 754 binary64 (double precision) on a PC with 2 $\mathrm{GHz}$ Intel Core i7-3667U Processor, so that $\mathbf{u}=2^{-53} \approx 1.11 \times 10^{-16}$.

For Algorithm 2.8, we adopt accurate matrix multiplication algorithms in $[\mathbf{8}$, 11, 16]. From $[\mathbf{1 1}]$, the computations are automatically parallelized in BLAS and 
LAPACK. We implement an algorithm for accurate matrix multiplication satisfying (4), which is based on Algorithm 2.1-2.6.

3.2.1. Results for extremely ill-conditioned matrices. We confirm our analysis of Algorithm 2.8 by some numerical experiments. As a stopping criterion for Algorithm 2.8, we set $\varepsilon_{t o l}=10^{-6}$. Define

$$
\widehat{G}^{(k)}:=\left(X_{1: m_{k-1}}^{(k-1)}\right)^{\top} A X_{1: m_{k-1}}^{(k-1)} .
$$

From our analysis, we can expect $\kappa\left(\widehat{G}^{(k)}\right)$ is reduced by a factor around $\alpha_{k}=\delta_{k} /\left\|\widehat{G}^{(k-1)}\right\|$ from (32) in each iteration until $\left\|I-\widehat{G}^{(k-1)}\right\|<\varepsilon_{t o l}$. Then $n \mathbf{u} \lesssim \alpha_{k} \lesssim n^{2} \mathbf{u}$. From Section 3.1, we regard $\kappa\left(\widehat{G}^{(k)}\right)$ as $\kappa\left(\widehat{G}^{(k)}\right) \approx\left(n^{2} \mathbf{u}\right)^{k} \kappa(A)$. However, from (31), $\delta_{k}$ indeed depends on the trace of $\widehat{G}^{(k-1)}$, i.e., $\delta_{k} \approx n \mathbf{u} \cdot \operatorname{tr}\left(\widehat{G}^{(k-1)}\right)$. In some cases, $\operatorname{tr}\left(\widehat{G}^{(k-1)}\right) \ll n\left\|\widehat{G}^{(k-1)}\right\|$ may occur. In this section, we define $\beta_{k}$ as

$$
\beta_{k}:=\frac{\operatorname{tr}\left(\widehat{G}^{(k-1)}\right)}{\left\|\widehat{G}^{(k-1)}\right\|} .
$$

Then

$$
\delta_{k} \approx n \mathbf{u} \cdot \beta_{k}\left\|\widehat{G}^{(k-1)}\right\|
$$

Moreover, we define $c_{k}$ as

$$
\begin{aligned}
& c_{1}:=\beta_{1} \\
& c_{k}:=c_{k-1} \cdot \beta_{k} \text { for } k \geq 2 .
\end{aligned}
$$

We observe how $\kappa\left(\widehat{G}^{(k)}\right)$ reduces in each iteration by a factor $n \mathbf{u} \beta_{k}$, as compared with $n^{2} u$.

First, we deal with the Higham's test matrices [3] on Matlab:

randsvd - Random matrix with pre-assigned singular values.

$\mathrm{A}=\operatorname{gallery}($ 'randsvd', $\mathrm{n}, \mathrm{kappa}$, mode $, \mathrm{kl}, \mathrm{ku}$ )

returns a banded (multidiagonal) random matrix of order $\mathrm{n}$ with 
TABLE 3.1. Results for Higham's test matrix with $n=2000$ and $\kappa(A) \approx 2.89 \cdot 10^{16}$.

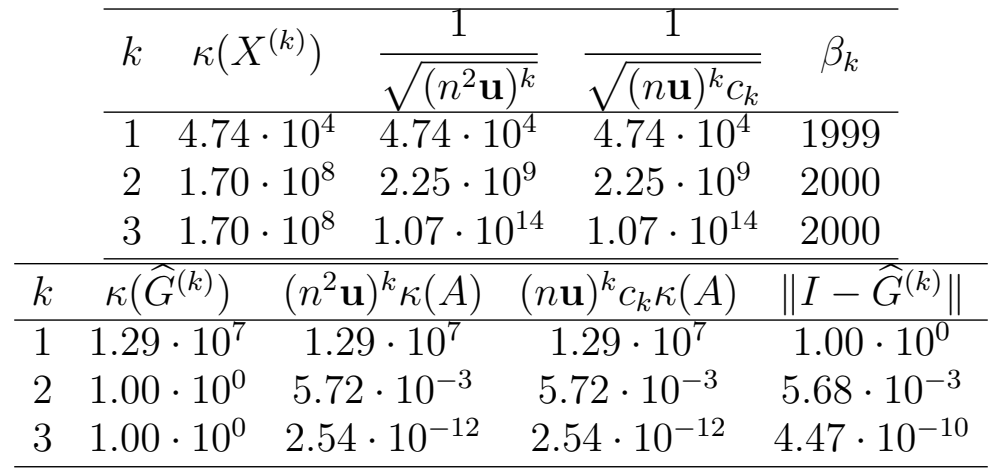

cond $(A)=$ kappa and singular values from the distribution mode. If $\mathrm{n}$ is a two-element vector, $\mathrm{A}$ is $\mathrm{n}(1)-\mathrm{by}-\mathrm{n}(2)$.

Mode may be one of the following values:

1: One large singular value.

2: One small singular value.

3: Geometrically distributed singular values (default).

4: Arithmetically distributed singular values.

5: Random singular values with uniformly distributed logarithm.

We generate matrices $A$ by $A=$ gallery('randsvd', $n, c n d, 3)$. We set $n=2000$ and cnd $=10^{16}$. Then $A \in \mathbb{F}^{2000 \times 2000}$ with $\kappa(A) \approx 2.89 \cdot 10^{16}$ is generated whose results are shown in Table 3.1.

Next, we deal with the Hilbert matrix. Due to rounding errors the condition number of floating-point Hilbert matrix is limited not far from $\mathbf{u}^{-1}$. Therefore we use the Hilbert matrix scaled by the least common multiple of the denominators as follows:

$$
H_{n}=\left(h_{i j}\right) \in \mathbb{F}^{n \times n} \quad \text { with } \quad h_{i j}:=\operatorname{lcm}(1,2, \cdots, 2 n-1) /(i+j-1) .
$$


TABLE 3.2. Results for scaled Hilbert matrix with $n=21$ and $\kappa(A) \approx$ $8.16 \cdot 10^{29}$.

\begin{tabular}{|c|c|c|c|c|c|c|}
\hline & $k$ & \multicolumn{2}{|c|}{$\kappa\left(X^{(k)}\right)$} & $\frac{1}{\sqrt{\left(n^{2} \mathbf{u}\right)^{k}}}$ & $\frac{1}{\sqrt{(n \mathbf{u})^{k} c_{k}}}$ & $\beta_{k}$ \\
\hline & 1 & $1.77 \cdot 1$ & & $4.42 \cdot 10^{6}$ & $1.77 \cdot 10^{7}$ & 1.3062 \\
\hline & 2 & $9.85 \cdot 10$ & & $1.95 \cdot 10^{13}$ & $9.95 \cdot 10^{13}$ & 12.8547 \\
\hline & 3 & $9.03 \cdot 10$ & & $8.61 \cdot 10^{19}$ & $4.52 \cdot 10^{20}$ & 17.9578 \\
\hline$k$ & & $\left.\widehat{G}^{(k)}\right)$ & $\left(n^{2}\right.$ & $\mathbf{u})^{k} \kappa(A)$ & $(n \mathbf{u})^{k} c_{k} \kappa(A)$ & $\left\|I-\widehat{G}^{(k)}\right\|$ \\
\hline 1 & 2.6 & $1 \cdot 10^{15}$ & & $19 \cdot 10^{16}$ & $2.60 \cdot 10^{15}$ & $1.00 \cdot 10^{0}$ \\
\hline 2 & 8.4 & $1 \cdot 10^{1}$ & & $15 \cdot 10^{3}$ & $8.24 \cdot 10^{1}$ & $9.88 \cdot 10^{-1}$ \\
\hline 3 & 1.0 & $0 \cdot 10^{0}$ & 1.1 & $0 \cdot 10^{-10}$ & $4.00 \cdot 10^{-12}$ & $4.09 \cdot 10^{-12}$ \\
\hline
\end{tabular}

Here $H_{n}$ is an integer matrix whose elements are exactly representable in binary64 floating-point numbers for $n \leq 21$ with $\kappa\left(H_{21}\right)=8.16 \cdot 10^{29}$. The results of Algorithm 2.8 for $A=H_{21}$ are shown in Table 3.2.

Moreover, we generate a test matrix with a specified condition number by $A:=$ $Q \Sigma Q^{\top} \in \mathbb{F}_{\left[\left[\log _{10}(c n d) / \mathbf{u}\right]\right]}^{n \times n}$, where $Q \in \mathbb{F}^{n \times n}$ is a random orthogonal matrix, $\Sigma:=$ $\operatorname{logspace}\left(1, \log _{10}(c n d), n\right)$ and $c n d$ is the condition number of $A$. We name this function testmatrix $(n, c n d)$. We also give the complete Matlab-code in Appendix. We set $n=100$, cnd $=10^{100}$. Then $A \in \mathbb{F}_{[7]}^{100 \times 100}$ with $\kappa(A) \approx 1.00 \cdot 10^{100}$ is generated, whose results are shown in Table 3.3. Finally, we deal with the random matrix, which is based on the function randmat in INTLAB [14], and surely generates symmetric and positive definite matrices. We name the function randmatsym $(n, c n d)$. This function use unit lower triangular $L$ and compute $A=L^{\top} A, A=A^{\top} A$ until $\kappa(A) \geq c n d$. We set $n=500$ and $c n d=10^{50}$. Then $A \in \mathbb{F}^{500 \times 500}$ with $\kappa(A) \approx 4.756 \cdot 10^{53}$ is generated, whose results are shown in Table 3.4. And, we set $n=1000$ with $\mathrm{cnd}=10^{100}$. Then $A \in \mathbb{F}^{1000 \times 1000}$ with $\kappa(A) \approx 8.297 \cdot 10^{102}$ is generated, whose results are shown in Table 3.5.

In all of the numerical experiments, it can be seen that the convergence speed of $\kappa\left(\widehat{G}^{(k)}\right)$ for $k \geq 2$ is about $\left(n^{2} \mathbf{u}\right)^{k} \kappa(A)$ during $\kappa\left(\widehat{G}^{(k)}\right)>\mathbf{u}^{-1}$, which is consistent with (62) in Analysis 2 as expected. However, for $k=1$, we can observe $\kappa\left(\widehat{G}^{(1)}\right)$ is relatively 
TABLE 3.3. Result for the test matrix with $n=100$ and $\kappa(A) \approx$ $1.00 \times 10^{100}$.

\begin{tabular}{cccccc}
\hline & $k$ & $\kappa\left(X^{(k)}\right)$ & $\frac{1}{\sqrt{\left(n^{2} \mathbf{u}\right)^{k}}}$ & $\frac{1}{\sqrt{(n \mathbf{u})^{k} c_{k}}}$ & $\beta_{k}$ \\
\hline & & $8.93 \cdot 10^{06}$ & $9.40 \cdot 10^{05}$ & $8.93 \cdot 10^{06}$ & 1.1083 \\
& 2 & $2.22 \cdot 10^{13}$ & $8.83 \cdot 10^{11}$ & $2.22 \cdot 10^{13}$ & 14.261 \\
& 3 & $4.02 \cdot 10^{19}$ & $8.30 \cdot 10^{17}$ & $4.02 \cdot 10^{19}$ & 26.927 \\
& 4 & $6.03 \cdot 10^{25}$ & $7.80 \cdot 10^{23}$ & $6.03 \cdot 10^{25}$ & 39.316 \\
& 5 & $7.89 \cdot 10^{31}$ & $7.33 \cdot 10^{29}$ & $7.89 \cdot 10^{31}$ & 51.545 \\
& 6 & $9.30 \cdot 10^{37}$ & $6.89 \cdot 10^{35}$ & $9.29 \cdot 10^{37}$ & 63.657 \\
& 7 & $1.00 \cdot 10^{44}$ & $6.47 \cdot 10^{41}$ & $1.00 \cdot 10^{44}$ & 75.678 \\
& 8 & $7.10 \cdot 10^{49}$ & $6.08 \cdot 10^{47}$ & $1.01 \cdot 10^{50}$ & 87.624 \\
& 9 & $1.00 \cdot 10^{50}$ & $5.71 \cdot 10^{53}$ & $9.50 \cdot 10^{55}$ & 99.406 \\
\hline$k$ & $\kappa(\widehat{G}(k)$ & $\left(n^{2} \mathbf{u}\right)^{k} \kappa(A)$ & $(n \mathbf{u})^{k} c_{k} \kappa(A)$ & $\|I-\widehat{G}(k)\|$ \\
\hline 1 & $1.25 \cdot 10^{86}$ & $1.13 \cdot 10^{88}$ & $1.26 \cdot 10^{86}$ & $1.00 \cdot 10^{00}$ \\
2 & $2.02 \cdot 10^{73}$ & $1.28 \cdot 10^{76}$ & $2.03 \cdot 10^{73}$ & $1.00 \cdot 10^{00}$ \\
3 & $6.18 \cdot 10^{60}$ & $1.45 \cdot 10^{64}$ & $6.18 \cdot 10^{60}$ & $1.00 \cdot 10^{00}$ \\
4 & $2.75 \cdot 10^{48}$ & $1.64 \cdot 10^{52}$ & $2.75 \cdot 10^{48}$ & $1.00 \cdot 10^{00}$ \\
5 & $1.61 \cdot 10^{36}$ & $1.86 \cdot 10^{40}$ & $1.61 \cdot 10^{36}$ & $1.00 \cdot 10^{00}$ \\
6 & $1.16 \cdot 10^{24}$ & $2.11 \cdot 10^{28}$ & $1.16 \cdot 10^{24}$ & $1.00 \cdot 10^{00}$ \\
7 & $9.92 \cdot 10^{11}$ & $2.39 \cdot 10^{16}$ & $9.92 \cdot 10^{11}$ & $1.00 \cdot 10^{00}$ \\
8 & $1.98 \cdot 10^{00}$ & $2.70 \cdot 10^{04}$ & $9.85 \cdot 10^{-1}$ & $4.96 \cdot 10^{-1}$ \\
9 & $1.00 \cdot 10^{00}$ & $3.06 \cdot 10^{-8}$ & $1.11 \cdot 10^{-12}$ & $2.23 \cdot 10^{-12}$ \\
\hline
\end{tabular}

much less than $\left(n^{2} \mathbf{u}\right) \kappa(A)$. This difference is caused by regarding $\delta_{1} \approx n^{2} \mathbf{u}\|A\|$ instead of $\delta_{1} \approx n \mathbf{u} \cdot \operatorname{tr}(A)$. In fact, we observe $\beta_{1} \approx 1$ in Table 3.2 and Table 3.3. This means $\delta_{1} \approx n \mathbf{u}\|A\|$. In the same way in Table 3.4 and Table 3.5 , we can observe $\beta_{1} \approx 20$, which is much less than $n$. In Table 3.1 , we can observe $\beta_{1} \approx n$. This means $\delta_{1} \approx n^{2} \mathbf{u}\|A\|$. In our analysis, the perturbation $\delta_{k} \approx \alpha_{k}\left\|\widehat{G}^{(k-1)}\right\|$ causes an improvement of $X^{(k)}$ as a preconditioner by a factor $1 / \sqrt{\alpha_{k}}$. Therefore, $\kappa\left(\widehat{G}^{(k)}\right)$ is reduced by a factor around $\alpha_{k}$ in each iteration until $\kappa\left(\widehat{G}^{(k)}\right) \approx 1$. In Table 3.2, Table 3.4 and Table 3.5 , for $k=1, \delta_{1} \approx n \mathbf{u} \beta_{1}\|A\|$ causes an improvement of $X^{(1)}$ as a preconditioner by a factor $1 / \sqrt{n \mathbf{u} \beta_{1}}$ rather than $1 / \sqrt{n^{2} \mathbf{u}}$ in practice. Therefore, $\kappa\left(\widehat{G}^{(1)}\right)$ reduces by a factor $n \mathbf{u} \beta_{1}$ from $\kappa(A)$. For $k \geq 2$, we can observe $\beta_{k} \approx n$, so that $\delta_{k} \approx n^{2} \mathbf{u}\left\|\widehat{G}^{(k-1)}\right\|$. It means $n \mathbf{u} \beta_{k} \approx n^{2} \mathbf{u}$ as shown in the tables. 
TABLE 3.4. Result for Rump's random matrix with $n=500$ and $\kappa(A) \approx 4.756 \times 10^{53}$.

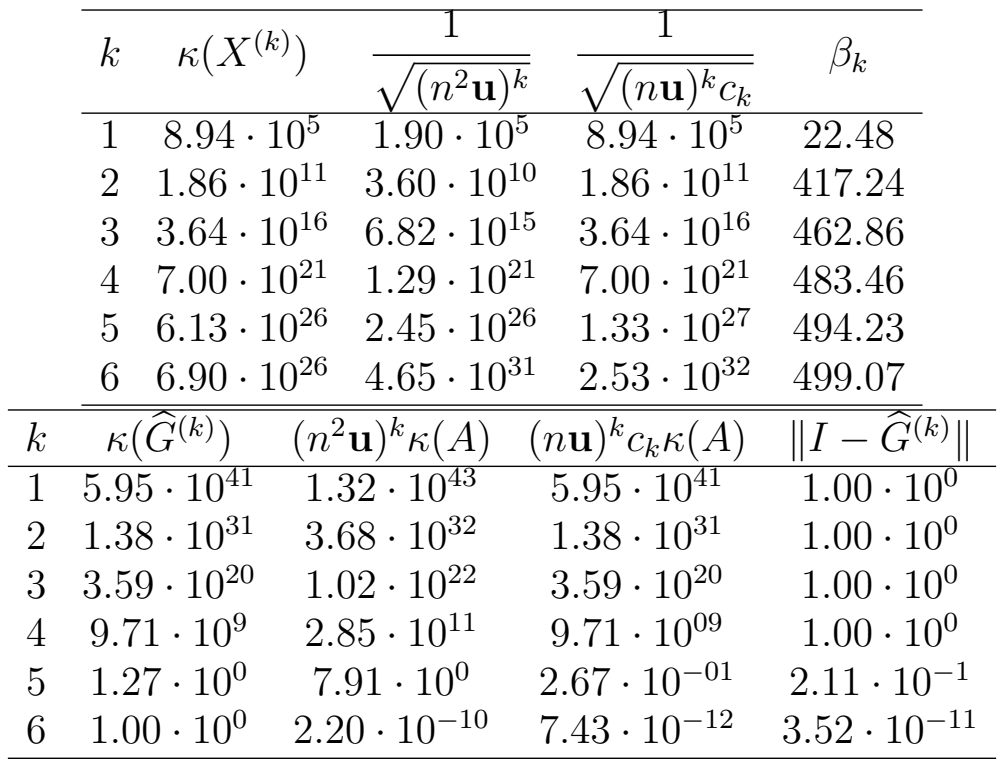


TABLE 3.5. Result for Rump's random matrix with $n=1000$ and $\kappa(A) \approx 8.297 \times 10^{102}$.

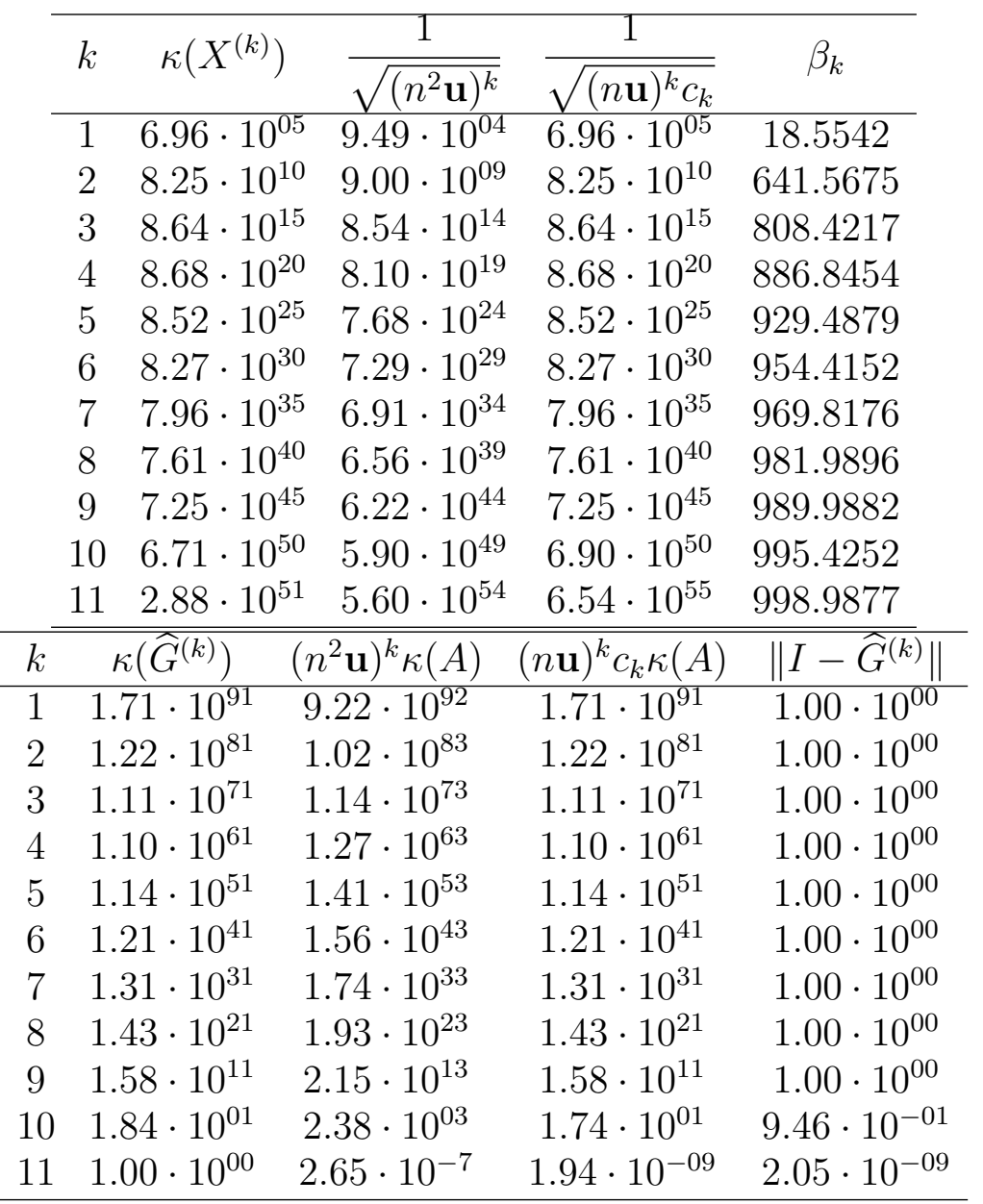


3.2.2. Comparison with long precision arithmetic. We present some numerical results comparing Algorithm 2.8 with a standard Cholesky factorization applying a diagonal shift by using long precision arithmetic such as GMP $[\mathbf{2}]$ and MPFR [5]. We measure computing time for verifying the positive definiteness of an input matrix. For this purpose, we use the Rump's corollary in [15].

Corollary 3.1 (Corollary 2.4 in $[\mathbf{1 5}]$ ). Let $\tilde{A} \in \mathbb{F}^{n \times n}$ be given with $\tilde{A}=A$ for $i \neq j$ and $\tilde{A} \leq A-c I$ where $c:=c_{n}^{\prime} \mathbf{u} \cdot \operatorname{tr}(A) \in \mathbb{F}$ for $i=j$. If the floating-point Cholesky factorization applied to $\tilde{A}$ runs to completion, then $A$ is positive definite.

We adopt the following Matlab routine mpfr_chol_spd, in which computational precision $2^{-d}$ is doubled per iteration with starting at $d=53$ :

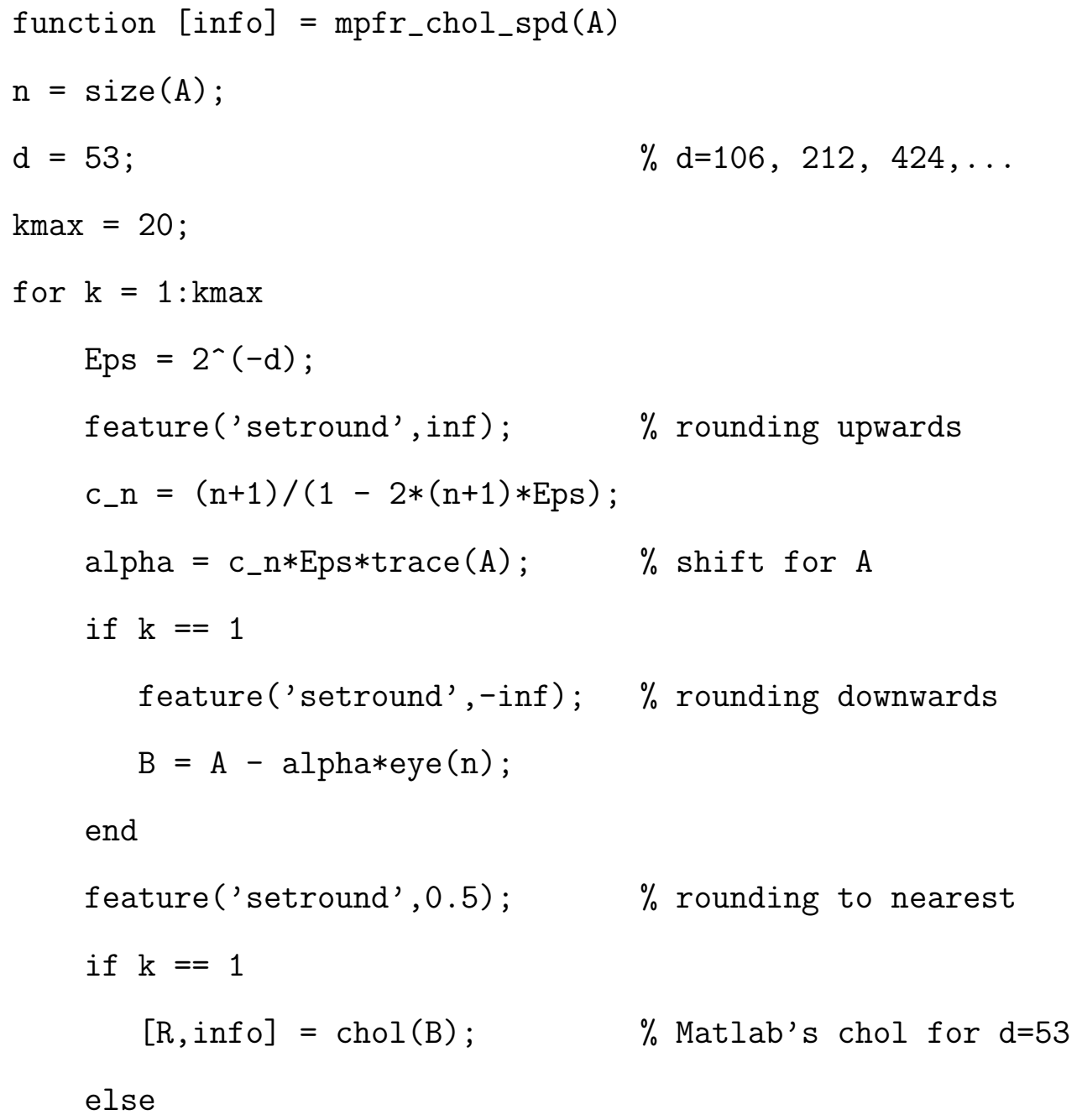




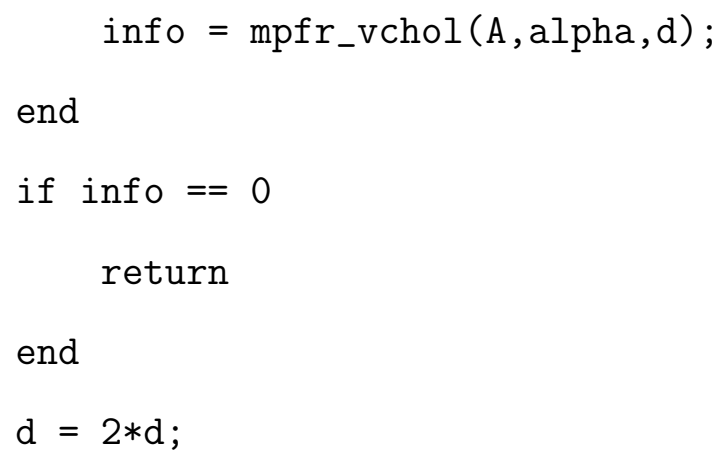

In this function, we use GMP [2] and MPFR [5], which are widely used as fast and standard long precision libraries. We implement Cholesky factorization using MPFR as Matlab's MEX function mpfr_vchol without any parallelization. In mpfr_vchol, the double precision input $A$ and the shift alpha is converted to $d$-bit precision numbers. Then $d$-bit precision arithmetic is internally used. If Cholesky factorization runs to completion, then info $=0$ as the result of mpfr_vchol is obtained. We give the complete C-code mpfr_vchol in Appendix. Note that only one core is used for mpfr_chol_spd, so that its computing time may be halved in an ideal case.

As mentioned before, Algorithm 2.8 uses for accurate matrix multiplication, which is based on the algorithms for accurate sum and dot product proposed in $[\mathbf{8}, \mathbf{1 8}, \mathbf{1 9}]$. Note that the resultant $m_{1}$ where the number of iterations for Algorithm 2.8 coincides with (96). From Theorem 3.2 in Chpter 2, we set $\varepsilon_{t o l}=1$.

Now we observe the computing time for two examples. We again use Rump matrix [13]. Here, $t_{1}$ and $t_{2}$ denote the computing time for Algorithm 2.8 and that for mpfr_chol_spd, respectively.

First, we fix $n=2000$ and vary anticipated condition numbers $c$ d from $10^{20}$ to $10^{100}$. The results are displayed in Table 3.6. As can be seen, $t_{1}$ gradually increases according to the condition numbers. For more than $c$ nd $=10^{60}, t_{1}$ is from 2 to 3 times larger than $t_{2}$. This is because the computational cost of the Algorithm 2.8 is strongly dependent on that of an algorithm for accurate matrix multiplication which 
TABLE 3.6. Result for Rump matrix randmatsym $(n, c n d)$ with $n=$ 2000 and several anticipated condition numbers $c n d\left(t_{i}\right.$ : elapsed time [sec], $m_{1}$ : number of iterations for Algorithm 2.8, $d$ : computational precision for MPFR as $2^{-d}$ ).

\begin{tabular}{ccccccc}
\hline & & \multicolumn{2}{c}{ Algorithm 2.8 } & & \multicolumn{2}{c}{ mpfr_chol_spd } \\
\cline { 3 - 4 } cnd & $\kappa(A)$ & $t_{1}$ & $m_{1}$ & & $t_{2}$ & $\frac{d}{53}$ \\
\hline $10^{20}$ & $8.4424 \cdot 10^{21}$ & 70.731 & 3 & & 126.214 & 2 \\
$10^{40}$ & $8.0047 \cdot 10^{42}$ & 199.59 & 5 & & 290.470 & 4 \\
$10^{60}$ & $1.4189 \cdot 10^{65}$ & 436.04 & 7 & & 294.928 & 4 \\
$10^{80}$ & $5.2528 \cdot 10^{83}$ & 749.46 & 9 & & 520.633 & 8 \\
$10^{100}$ & $5.9425 \cdot 10^{107}$ & 1431.87 & 12 & & 530.825 & 8 \\
\hline
\end{tabular}

TABLE 3.7. Result for Rump matrix randmatsym $(n, c n d)$ with several $n$ and $\kappa(A) \approx 10^{19}\left(t_{i}\right.$ : elapsed time [sec], $m$ : number of iterations for Algorithm 2.8, $d$ : computational precision for MPFR as $2^{-d}$ ).

\begin{tabular}{cccccc}
\hline & \multicolumn{2}{c}{ Algorithm 2.8} & & \multicolumn{2}{c}{ mpfr_chol_spd } \\
\cline { 2 - 3 } \cline { 5 - 6 }$n$ & $t_{1}$ & $m_{1}$ & & $t_{2}$ & $\frac{d}{53}$ \\
\hline 500 & 1.02 & 2 & & 2.096 & 2 \\
1000 & 4.60 & 2 & & 16.219 & 2 \\
2000 & 28.08 & 2 & & 126.525 & 2 \\
3000 & 224.44 & 3 & & 424.724 & 2 \\
4000 & 496.18 & 3 & & 975.986 & 2 \\
5000 & 1015.08 & 3 & & 2003.862 & 2 \\
\hline
\end{tabular}

requires $\mathcal{O}\left(m^{3} n^{3}\right)$ flops, while that of mpfr_vchol requires $\mathcal{O}\left(n^{3}\right)$ with large constant per iteration. Namely, in case of $A$ being extremely ill-conditioned, i.e. $\kappa(A) \geq 10^{60}$, mpfr_chol_spd has an advantage over Algorithm 2.8 in terms of computational cost.

Next, we fix $c n d=10^{19}$ and vary $n$ from 500 to 5000 . The results are displayed in Table 3.7. As expected, $t_{1}$ is much smaller than $t_{2}$. We observe that $t_{2}$ increases in $\mathcal{O}\left(n^{3}\right)$ according to the dimension $n$, while $t_{1}$ does not in $\mathcal{O}\left(m^{3} n^{3}\right)$ for smaller $n$. Note that the BLAS and the LAPACK routines adopted in Matlab are provided by Intel Math Kernel Library are highly optimized. In particular, in the case of $n \geq 4000$, we expect that they achieve near-peak performance. It turns out that Algorithm 2.8 brings out the computer performance to the maximum rather than mpfr_chol_spd. 



\section{Chapter 4}

\section{A MODIFIED ALGORITHM FOR}

\section{ACCURATE INVERSE ChOlesky \\ FACTRIZATION}


In this chapter, we consider the accuracy on $X_{1: m_{k}}^{(k)} \in \mathbb{F}_{\left[m_{k}\right]}^{n \times n}$ provided by Algorithm 2.8. In Section 4.1, a modified version of the algorithm is proposed. It is explained that the proposed algorithm gives more accurate results than the original one by a numerical analysis in Section 4.2. In Section 4.3, Numerical evidence is also shown.

\subsection{Proposed ALgorithm}

From (90) in Analysis 2 in Section 3.1, it hods that

$$
\left\|I-X_{1: m_{k}}^{(k) \top} A X_{1: m_{k}}^{(k)}\right\| \approx \alpha_{k} \mu
$$

where $\mu:=\min \left(\kappa\left(\left(X_{1: m_{k-1}}^{(k-1)}\right)^{\top} A X_{1: m_{k-1}}^{(k-1)}\right), \alpha_{k}^{-1}\right)$. From (32), we have $n \mathbf{u} \lesssim \alpha_{k} \lesssim n^{2} \mathbf{u}$. If $\kappa\left(\left(X_{1: m_{k-1}}^{(k-1)}\right)^{\top} A X_{1: m_{k-1}}^{(k-1)}\right)>\alpha_{k}^{-1}$, then the definition of $\mu$ implies $\mu=\alpha_{k}^{-1}$ and

$$
\left\|I-X_{1: m_{k}}^{(k) \top} A X_{1: m_{k}}^{(k)}\right\| \approx 1 .
$$

Otherwise, $\mu=\kappa\left(\left(X_{1: m_{k-1}}^{(k-1)}\right)^{\top} A X_{1: m_{k-1}}^{(k-1)}\right)$ and

$$
\left\|I-X_{1: m_{k}}^{(k) \top} A X_{1: m_{k}}^{(k)}\right\|<1 .
$$

Furthermore, in the best case as $\kappa\left(\left(X_{1: m_{k-1}}^{(k-1)}\right)^{\top} A X_{1: m_{k-1}}^{(k-1)}\right)=1$, we have $\mu=1$ and

$$
\left\|I-X_{1: m_{k}}^{(k) \top} A X_{1: m_{k}}^{(k)}\right\| \approx \alpha_{k} .
$$

Therefore, it holds that

$$
\alpha_{k} \approx\left\|I-X_{1: m_{k}}^{(k) \top} A X_{1: m_{k}}^{(k)}\right\|<1
$$

The reason why $\alpha_{k}$ is the lower bound is due to the diagonal shift (98) in Algorithm 2.8. To overcome (97), we add a condition such that Cholesky factorization never breaks down without a diagonal shift. 
For a symmetric matrix $A=\left(a_{i j}\right) \in \mathbb{F}^{n \times n}$ with $a_{i i}>0$ for all $i$, the following algorithm calculates an upper triangular matrix $X_{1: m_{M}}^{(M)} \mathbb{F}_{\left[m_{k}\right]}^{n \times n}$ where $M:=k+1$ for some $k \in \mathbb{N}$ such that $\left\|X_{1: m_{M}}^{(M) T} A X_{1: m_{M}}^{(M)}-I\right\| \approx \mathbf{u}$. 
Algorithm 4.1. Accurate inverse Cholesky factorization, modified version.

function $\left[X_{1: m_{M}}^{(M)}\right]=$ accchol_mod $(A)$

$k=0, \quad G^{(0)}:=A_{1: 1}, \quad E^{(0)}:=O, \quad X_{1: 1}^{(0)}:=I$

repeat

$$
k=k+1
$$

Compute $S^{(k)} \in \mathbb{F}^{n \times n}$ with

$$
\left\{\begin{array}{l}
S_{i i}^{(k)}=f l_{\triangle}\left(G_{i i}^{(k-1)}+\left\|E^{(k-1)}\right\|_{\infty}\right) \\
S_{i j}^{(k)}=G_{i j}^{(k-1)} \text { for } i \neq j .
\end{array} .\right.
$$$$
\delta_{k}:=\operatorname{shift}\left(S^{(k)}\right)
$$$$
R^{(k)}:=\operatorname{chol}\left(f\left(S^{(k)}+\delta_{k} I\right)\right)
$$

$T^{(k)}:=\operatorname{triinv}\left(R^{(k)}\right)$

$$
m_{k}:=\left\lceil\frac{k}{2}\right\rceil+1
$$$$
X_{1: m_{k}}^{(k)}:=\left\{X_{1: m_{k-1}}^{(k-1)} T^{(k)}\right\}_{m_{k}}^{m_{k}}
$$$$
\left\langle G^{(k)}, E^{(k)}\right\rangle:=\left\{X_{1: m_{k}}^{(k) T} A X_{1: m_{k}}^{(k)}\right\}_{k+1}^{1}
$$

$\beta:=f_{\nabla}\left(\min \left(G_{i i}^{(k)}-r_{i}\left(G^{(k)}\right)\right)\right)$

$$
\mu:=f l_{\triangle}\left(c_{n}^{\prime} \mathbf{u} \cdot \operatorname{tr}\left(G^{(k)}\right)\right) \quad \% c_{n}^{\prime}:=\frac{n+1}{1-2(n+1) \mathbf{u}}
$$

until $\quad \beta>\max \left(\mu,\left\|E^{(k)}\right\|_{\infty}\right)$

$$
\begin{aligned}
& R^{(M)}:=\operatorname{chol}\left(G^{(M-1)}\right) \quad \% M:=k+1 \\
& T^{(M)}:=\operatorname{triinv}\left(R^{(M)}\right) \\
& m_{M}:=\left\lceil\frac{k+1}{2}\right\rceil+1 \\
& X_{1: m_{M}}^{(M)}:=\left\{X_{1: m_{k}}^{(M-1)} T^{(M)}\right\}_{m_{M}}^{m_{M}}
\end{aligned}
$$


In the following, we explain how to determine (100). For readability, $G^{(k)}, X_{1: m_{k}}^{(k)}$ and $X_{1: m_{M}}^{(M)}$ where $M:=k+1$ are abbreviated to $G, X$ and $X^{\prime}$, respectively. Let $R^{\prime}$ be a computed Cholesky factor of $G$, i.e., $R^{\prime}=\operatorname{chol}(G)$. Define

$$
\begin{aligned}
\Delta_{1} & :=G-X^{\top} A X, \\
\Delta_{2} & :=G-R^{\prime \top} R^{\prime}, \\
T & :=\operatorname{triinv}\left(R^{\prime}\right), \\
\Delta_{3} & :=X^{\prime}-X T,
\end{aligned}
$$

By the definition (102), it is necessary to satisfy

$$
\lambda_{n}\left(G-\Delta_{2}\right)>0
$$

By (15) and a Weyl's theorem (e.g. Corollary 4.9 in [21]) for (102), it holds that

$$
\lambda_{n}\left(G-\Delta_{2}\right) \geq \lambda_{n}(G)-\left\|\Delta_{2}\right\|, \quad\left\|\Delta_{2}\right\| \leq c_{n}^{\prime} \mathbf{u} \cdot \operatorname{tr}(G) .
$$

The Gerschgorin's circle theorem implies

$$
\lambda_{k}(G) \in \bigcup_{i=1}^{n}\left\{x \in \mathbb{R}:\left|x-G_{i i}\right| \leq r_{i}(G)\right\}, \quad k=1,2, \ldots, n
$$

and

$$
\lambda_{n}(G) \geq \beta \quad \text { where } \quad \beta:=\min \left(G_{i i}-r_{i}(G)\right)
$$

From (105) and (106), we have

$$
\lambda_{n}\left(G-\Delta_{2}\right) \geq \beta-\left\|\Delta_{2}\right\| .
$$


Moreover, to ensure the positive definiteness of $A$ with taking care of the rounding errors, it is necessary to satisfy

$$
\lambda_{n}\left(X^{\top} A X\right)>0 .
$$

By a Weyl's theorem (e.g. Corollary 4.9 in [21]) for (101), it holds that

$$
\lambda_{n}\left(X^{\top} A X\right) \geq \lambda_{n}(G)-\left\|\Delta_{1}\right\|
$$

From (106), we have

$$
\lambda_{n}\left(X^{\top} A X\right) \geq \beta-\left\|\Delta_{1}\right\|
$$

Therefore, combining (107) and (108), the sufficient condition to make $\operatorname{chol}(G)$ run to completion and to ensure the positive definiteness of $A$ is

$$
\beta>\max \left(\left\|\Delta_{1}\right\|,\left\|\Delta_{2}\right\|\right)
$$

\subsection{Analysis OF ACCURACY ON $X$}

The target of this subsection is to explain that $\left\|X^{\prime \top} A X^{\prime}-I\right\| \approx \mathbf{u}$ if $G^{(k)}$ satisfy (109) at the $k$-th iteration. For later use, we present the following observation.

Observation 4.1. Let $A=\left(a_{i j}\right) \in \mathbb{F}^{n \times n}$ be symmetric and positive definite. Split $A=I+E$. Let $E_{U}$ be the strictly upper triangular part of $E$. Suppose $\|E\| \leq \alpha \ll 1$. Let a triangular matrix $R=\left(r_{i j}\right) \in \mathbb{F}^{n \times n}$ be a computed Cholesky factor of A, i.e., $R=\operatorname{chol}(A)$. We use Algorithm 2.7. Then

$$
R \approx I+E_{U}, \quad\left\|E_{U}\right\| \leq \alpha \quad \text { and } \quad\left\|A-R^{\top} R\right\| \lesssim \mathbf{u}+n \alpha^{2} .
$$

Argument First, we estimate diagonal and off-diagonal elements of $R$, respectively. Here $\mathrm{fl}(\cdot)$ means that arithmetic inside the parenthesis is done in binary64 
floating point arithmetic. It follows that

$$
\begin{aligned}
r_{j j} & =\mathrm{fl}\left(\left(a_{j j}-\sum_{k=1}^{j-1} r_{k j}^{2}\right)^{\frac{1}{2}}\right)=(1+\epsilon)\left(a_{j j}-\epsilon_{1}\right)^{\frac{1}{2}} \\
& =(1+\epsilon)\left(1+\epsilon^{\prime}\right)^{\frac{1}{2}} \approx 1+\frac{1}{2} \epsilon^{\prime} \\
& |\epsilon| \leq \mathbf{u}, \quad\left|\epsilon_{1}\right| \leq(n-1) \alpha^{2}, \quad\left|\epsilon^{\prime}\right| \approx \alpha
\end{aligned}
$$

and

$$
r_{i j}=\mathrm{fl}\left(\left(a_{i j}-\sum_{k=1}^{i-1} r_{k i} r_{k j}\right) / r_{i i}\right)=a_{i j}+\epsilon_{2} \quad \text { for } \quad i<j, \quad\left|\epsilon_{2}\right| \approx \alpha^{2}
$$

Thus, we have $r_{j j} \lesssim 1+\frac{1}{2} \alpha$ and $\left|r_{i j}\right| \lesssim \alpha$. Then $R \approx I+E_{U}$.

Next, we estimate $\left\|A-R^{\top} R\right\|$. On the off-diagonal elements, it holds [3, p.197] that

$$
\left|a_{i j}-\sum_{k=1}^{i} r_{k i} r_{k j}\right| \leq \gamma_{i} \sum_{k=1}^{i}\left|r_{k i}\right|\left|r_{k j}\right| \quad \text { where } \quad \gamma_{i}=\frac{i \mathbf{u}}{1-i \mathbf{u}}
$$

Here, from (110) and (111), we have

$$
\begin{aligned}
\left|a_{i j}-\sum_{k=1}^{i} r_{k i} r_{k j}\right| & \leq \gamma_{i}\left(\sum_{k=1}^{i-1}\left|r_{k i}\right|\left|r_{k j}\right|+\left|r_{i i}\right|\left|r_{i j}\right|\right) \\
& \approx(n-1) \mathbf{u}\left((n-2) \alpha^{2}+\left(1+\frac{1}{2} \alpha\right) \alpha\right) \approx n \mathbf{u} \alpha .
\end{aligned}
$$

From $(110), r_{j j}^{2}=(1+\epsilon)^{2}\left(a_{j j}-\epsilon_{1}\right) \approx(1+2 \epsilon) a_{j j}$. Hence, it holds that

$$
\begin{aligned}
\left|a_{j j}-\sum_{k=1}^{j} r_{k j}^{2}\right| & \leq\left|a_{j j}-r_{j j}^{2}\right|+\sum_{k=1}^{j-1} r_{k j}^{2} \approx\left|a_{j j}-(1+2 \epsilon) a_{j j}\right|+(n-1) \alpha^{2} \\
& \approx 2|\epsilon| a_{j j}+n \alpha^{2} \approx \mathbf{u}+n \alpha^{2}
\end{aligned}
$$

Therefore, we have $\left\|A-R^{\top} R\right\| \approx n \mathbf{u} \alpha+\mathbf{u}+n \alpha^{2} \approx \mathbf{u}+n \alpha^{2}$.

REMARK 8. If $n \alpha^{2}<\mathbf{u}$, then $\left\|A-R^{\top} R\right\| \approx \mathbf{u}$. 
Observation 4.2. Let $R$ and $E_{U}$ be defined as in Observation 4.1. Let $T:=$ $\operatorname{triinv}(R)$. Then

$$
T \approx I-E_{U} \text { and }\|T\| \approx 1
$$

Argument From Observation 4.1, it follows that $R \approx I+E_{U}$ and $\left\|E_{U}\right\| \ll 1$. Then

$$
T=\left(I+E_{U}\right)^{-1}=I-E_{U}+E_{U}^{2}-E_{U}^{3}+\cdots \approx I-E_{U}
$$

and

$$
\|T\| \approx 1
$$

Observation 4.3. Let $R, T$ and $E_{U}$ be defined as in Observations 4.1 and 4.2. Then

$$
\left\|I-T^{\top} R^{\top} R T\right\| \approx \alpha^{2} .
$$

Argument From Observations 4.1 and 4.2, it follows that

$$
R T \approx\left(I+E_{U}\right)\left(I-E_{U}\right)=I-E_{U}^{2}
$$

and

$$
\left\|I-T^{\top} R^{\top} R T\right\| \approx\left\|I-\left(I-E_{U}^{2}\right)^{\top}\left(I-E_{U}^{2}\right)\right\| \approx\left\|E_{U}^{2}\right\| \leq \alpha^{2} .
$$

Next, we estimate $\left\|I-X^{\prime \top} A X^{\prime}\right\|$ by the use of numerical error analysis.

ANAlysis 3. We utilize (101)-(104). Assume that $A \in \mathbb{F}^{n \times n}$ is positive definite, $(n+1)(n+3) \mathbf{u}<1$ and we can use high precision computations for dot product which 
satisfies (4). Assume $X$ is nearly the best case as

$$
\kappa\left(X^{\top} A X\right) \approx 1 \text { and }\left\|I-X^{\top} A X\right\| \approx \alpha<1 .
$$

We analyze to show

$$
\left\|I-X^{\prime \top} A X^{\prime}\right\| \approx \mathbf{u}+n \alpha^{2} .
$$

Argument From Analysis 2 in Chapter 3, we have

$$
\begin{aligned}
& \left\|X^{\top} A X\right\| \approx 1, \\
& \|X\| \lesssim\left((n \mathbf{u})^{k}\|A\|\right)^{-\frac{1}{2}} \text { and } \\
& \left\|X^{-1}\right\| \approx\|A\|^{\frac{1}{2}} .
\end{aligned}
$$

The computation of $X^{\prime}$ in $m_{M}$-fold working precision gives

$$
\left\|\Delta_{3}\right\| \leq \mathbf{u}^{\left\lceil\frac{k+1}{2}\right\rceil+1}\|X\|\|T\| \lesssim \mathbf{u}^{\left\lceil\frac{k+1}{2}\right\rceil+1}\left(\frac{(n \mathbf{u})^{-k}}{\|A\|}\right)^{\frac{1}{2}} \approx u\|A\|^{-\frac{1}{2}} .
$$

Then

$$
\begin{aligned}
\left\|I-X^{\prime \top} A X^{\prime}\right\| & =\left\|I-\left(X T+\Delta_{3}\right)^{\top} A\left(X T+\Delta_{3}\right)\right\| \\
& \leq\left\|I-T^{\top} X^{\top} A X T\right\|+2\left\|T^{\top} X^{\top} A \Delta_{3}\right\|+\left\|\Delta_{3}^{\top} A \Delta_{3}\right\| .
\end{aligned}
$$

Here

$$
\begin{aligned}
\left\|I-T^{\top} X^{\top} A X T\right\| & =\left\|\left(I-T^{\top} G T\right)-T^{\top} \Delta_{1} T\right\| \\
& =\left\|\left(I-T^{\top}\left(R^{\prime \top} R^{\prime}+\Delta_{2}\right) T\right)-T^{\top} \Delta_{1} T\right\| \\
& \leq\left\|I-T^{\top} R^{\prime \top} R^{\prime} T\right\|+\left\|T^{\top} \Delta_{2} T\right\|+\left\|T^{\top} \Delta_{1} T\right\| .
\end{aligned}
$$


From Observation 4.3, we have

$$
\left\|I-T^{\top} R^{\prime \top} R^{\prime} T\right\| \approx \alpha^{2} .
$$

The computation of $X^{\top} A X$ by $\left\{X^{\top} A X\right\}_{k+1}^{1},(4),(113)$ and (114) imply

$$
\begin{aligned}
\left\|\Delta_{1}\right\| & \leq \varphi(\mathbf{u})\left\|X^{\top} A X\right\|+\varphi\left(\mathbf{u}^{k+1}\right)\left\|X^{\top}\right\|\|A\|\|X\| \\
& \lesssim \mathbf{u} \cdot 1+\mathbf{u}^{\mathbf{k}+\mathbf{1}} \cdot\left((n \mathbf{u})^{\mathbf{k}}\right)^{-\mathbf{1}} \approx \mathbf{u}
\end{aligned}
$$

From Observation 4.1, we have

$$
\left\|\Delta_{2}\right\| \approx \mathbf{u}+n \alpha^{2}
$$

From Observation 4.2, (120) and (121), it follows that

$$
\left\|T^{\top} \Delta_{2} T\right\| \leq\|T\|^{2}\left\|\Delta_{2}\right\| \approx \mathbf{u}+n \alpha^{2}, \quad\left\|T^{\top} \Delta_{1} T\right\| \leq\|T\|^{2}\left\|\Delta_{1}\right\| \approx \mathbf{u} .
$$

Inserting (119) and (122) into (118) yields

$$
\left\|I-T^{\top} X^{\top} A X T\right\| \approx \alpha^{2}+\mathbf{u}+n \alpha^{2}+\mathbf{u} \approx n \alpha^{2}+\mathbf{u}
$$

Moreover, we estimate $\left\|T^{\top} X^{\top} A \Delta_{3}\right\|$ and $\left\|\Delta_{3}^{\top} A \Delta_{3}\right\|$ in (117). By Observation 4.2, (115), (116) and (120), it holds

$$
\begin{aligned}
\left\|T^{\top} X^{\top} A \Delta_{3}\right\| & =\left\|T^{\top} X^{\top} A X X^{-1} \Delta_{3}\right\|=\left\|T^{\top}\left(G-\Delta_{1}\right) X^{-1} \Delta_{3}\right\| \\
& \leq\|T\|\left(\|G\|+\left\|\Delta_{1}\right\|\right)\left\|X^{-1}\right\|\left\|\Delta_{3}\right\| \approx \mathbf{u}
\end{aligned}
$$

and

$$
\left\|\Delta_{3}^{\top} A \Delta_{3}\right\| \leq\left\|\Delta_{3}\right\|^{2}\|A\| \approx \mathbf{u}^{3}
$$


Inserting (123)-(125) into (117) implies

$$
\left\|I-X^{\prime \top} A X^{\prime}\right\| \lesssim \mathbf{u}+n \alpha^{2}+\mathbf{u}+\mathbf{u}^{3} \approx \mathbf{u}+n \alpha^{2}
$$

which explains (112).

If $n \alpha^{2} \approx n^{5} \mathbf{u}^{2}<\mathbf{u}$ satisfies, then

$$
\left\|I-X^{\prime \top} A X^{\prime}\right\| \approx \mathbf{u}
$$

REMARK 9. Here, $n \alpha^{2}$ in (126), is an upper bound of the rounding errors. As we will see in Subsection 4.3, Algorithm 4.1 gives $\left\|I-X^{\prime \top} A X^{\prime}\right\| \approx \mathbf{u}$ for larger $n$ such that $n \alpha^{2}>\mathbf{u}$ in practice.

4.2.1. Computational complexity. We assume accurate dot product based on the algorithm in $[\mathbf{8}, \mathbf{1 1}, \mathbf{1 6}]$ is used in Algorithm 4.1. It requires $\mathcal{O}\left(k^{2} n^{3}\right)$ flops to compute the matrix multiplication $X^{(k-1)} T^{(k)}$ and $X^{(k) T} A X^{(k)}$ and $\mathcal{O}\left(n^{3}\right)$ flops to compute the rest of the algorithm at the $k$-th iteration. If the iterations stop at $k=m_{2}=M-1$, then the computational cost of the part of Algorithm 4.1 becomes $\mathcal{O}\left(m_{2}^{3} n^{3}\right)$ flops since $\sum_{k=1}^{m_{2}} k^{2} n^{3}=\mathcal{O}\left(m_{2}^{3} n^{3}\right)$. After the iterations, it requires $\mathcal{O}\left(M^{2} n^{3}\right)$ flops to compute the matrix multiplication $X^{(M-1)} T^{(M)}$ and $\mathcal{O}\left(n^{3}\right)$ flops to compute the rest of the algorithm. Then, the total cost of Algorithm 4.1 becomes $\mathcal{O}\left(M^{3} n^{3}\right)$ flops. From (62), we can see that the number of iterations $m_{2}$ in Algorithm 4.1 is estimated as

$$
m_{2}=\left\lceil\frac{\log \kappa(A)}{\log \alpha}\right\rceil, \quad \text { and } \quad M=m_{2}+1
$$

We will confirm it in Section 4.3. 


\subsection{NumERICAL EXPERIMENTS}

In this section we present some numerical results. All computations are done on Matlab 2012b using IEEE standard 754 binary64 (double precision) on Mac OS X version 10.8 with Intel Core i7-3667U Processor, so that $\mathbf{u}=2^{-53} \approx 1.11 \times 10^{-16}$.

We present some numerical results for comparing the proposed algorithm (Algorithm 4.1) with the original one (Algorithm 2.8), in terms of both the accuracy on $X$ and measured computing time. For Algorithms 2.8 and 4.1, we adopt accurate matrix multiplication algorithms in $[\mathbf{1 1}]$. Then the computations are automatically parallelized in BLAS and LAPACK. Define

$$
\widehat{G}^{(k)}:=\left(X_{1: m_{k-1}}^{(k-1)}\right)^{\top} A X_{1: m_{k-1}}^{(k-1)} .
$$

First, we observe some numerical results in terms of the accuracy on $X$. From (97), the lower bound of $\left\|I-X_{1: m_{k}}^{\top} A X_{1: m_{k}}\right\|$ in Algorithm 2.8 is about $n^{2} \mathbf{u}$. Thus, we set $\varepsilon_{t o l}=10^{\left\lceil\log _{10} n^{2} \mathbf{u}\right\rceil}$ as a stopping criterion for Algorithm 2.8. We deal with the Hilbert matrix as the first example. The results of Algorithm 2.8 for $A=H_{21}$ are shown in Table 4.1. As the second example, we deal with the Rump matrix [13] as an ill-conditioned matrix, which is based on the function randmat in INTLAB [14], and surely generates symmetric and positive definite matrices. We name the function randmatsym $(n, c n d)$. We set $n=500$ with $c n d=10^{50}$. Then $A \in \mathbb{F}^{500 \times 500}$ with $\kappa(A) \approx 4.756 \times 10^{53}$ is generated, and the results are shown in Table 4.2. Moreover, we set $n=1000$ with $c n d=10^{100}$. Then $A \in \mathbb{F}^{1000 \times 1000}$ with $\kappa(A) \approx 8.297 \times 10^{102}$ is generated, and the results are shown in Table 4.3.

Let $m_{1}$ and $m_{2}$ denote the number of iterations for Algorithm 2.8 and that for Algorithm 4.1, respectively. Put $M=m_{2}+1$. On Algorithm 2.8, it can be seen from Table 4.1 - Table 3.5 that $\kappa\left(\widehat{G}^{(k)}\right)$ is reduced by a factor around $n^{2} \mathbf{u}$ in each iteration until $\left\|I-\widehat{G}^{\left(m_{1}\right)}\right\| \approx \alpha$, which is consistent with (97) as expected. Moreover, we can observe that $\left\|I-\widehat{G}^{\left(m_{1}\right)}\right\|$ in Algorithm 2.8 increases according to the dimension $n$, 
TABLE 4.1. Results for scaled Hilbert matrix $\kappa(A) \approx 8.16 \cdot 10^{29}$ and $\varepsilon_{t o l}=10^{-13}$.

\begin{tabular}{cccccc}
\hline & & & Algorithm 2.8 & & Algorithm 4.1 \\
$k$ & $\kappa\left(\widehat{G}^{(k)}\right)$ & $\left(n^{2} \mathbf{u}\right)^{k} \kappa(A)$ & $\left\|I-\widehat{G}^{(k)}\right\|$ & & $\left\|I-\widehat{G}^{(k)}\right\|$ \\
\hline 1 & $2.61 \cdot 10^{15}$ & $4.19 \cdot 10^{16}$ & $1.00 \cdot 10^{00}$ & & $1.00 \cdot 10^{00}$ \\
2 & $8.35 \cdot 10^{01}$ & $2.15 \cdot 10^{03}$ & $9.88 \cdot 10^{-01}$ & & $9.88 \cdot 10^{-01}$ \\
3 & $1.00 \cdot 10^{00}$ & $1.10 \cdot 10^{-10}$ & $4.04 \cdot 10^{-12}$ & & $4.04 \cdot 10^{-12}$ \\
4 & $1.00 \cdot 10^{00}$ & $5.65 \cdot 10^{-24}$ & $5.13 \cdot 10^{-14}$ & & $3.79 \cdot 10^{-16}$ \\
\hline
\end{tabular}

TABLE 4.2. Result for Rump matrix with $n=500, \kappa(A) \approx 4.756 \times 10^{53}$ and $\varepsilon_{t o l}=10^{-10}$.

\begin{tabular}{|c|c|c|c|c|}
\hline & & & Algorithm 2.8 & Algorithm 4.1 \\
\hline$k$ & $\kappa\left(\widehat{G}^{(k)}\right)$ & $\left(n^{2} \mathbf{u}\right)^{k} \kappa(A)$ & $\left\|I-\widehat{G}^{(k)}\right\|$ & $\left\|I-\widehat{G}^{(k)}\right\|$ \\
\hline 1 & $5.95 \cdot 10^{41}$ & $1.32 \cdot 10^{43}$ & $1.00 \cdot 10^{00}$ & $1.00 \cdot 10^{00}$ \\
\hline 2 & $1.38 \cdot 10^{31}$ & $3.68 \cdot 10^{32}$ & $1.00 \cdot 10^{00}$ & $1.00 \cdot 10^{00}$ \\
\hline 3 & $3.59 \cdot 10^{20}$ & $1.02 \cdot 10^{22}$ & $1.00 \cdot 10^{00}$ & $1.00 \cdot 10^{00}$ \\
\hline 4 & $9.71 \cdot 10^{09}$ & $2.85 \cdot 10^{11}$ & $1.00 \cdot 10^{00}$ & $1.00 \cdot 10^{00}$ \\
\hline 5 & $1.27 \cdot 10^{00}$ & $7.91 \cdot 10^{00}$ & $2.11 \cdot 10^{-01}$ & $2.11 \cdot 10^{-01}$ \\
\hline 6 & $1.00 \cdot 10^{00}$ & $2.20 \cdot 10^{-10}$ & $3.52 \cdot 10^{-11}$ & $3.88 \cdot 10^{-16}$ \\
\hline
\end{tabular}

TABLE 4.3. Result for Rump matrix with $n=1000, \kappa(A) \approx 8.297 \times$ $10^{102}$ and $\varepsilon_{t o l}=10^{-9}$.

\begin{tabular}{|c|c|c|c|c|}
\hline & & & Algorithm 2.8 & Algorithm 4.1 \\
\hline$k$ & $\kappa\left(\widehat{G}^{(k)}\right)$ & $\left(n^{2} \mathbf{u}\right)^{k} \kappa(A)$ & $\left\|I-\widehat{G}^{(k)}\right\|$ & $\left\|I-\widehat{G}^{(k)}\right\|$ \\
\hline 1 & $1.71 \cdot 10^{91}$ & $9.22 \cdot 10^{92}$ & $1.00 \cdot 10^{00}$ & $1.00 \cdot 10^{00}$ \\
\hline 2 & $1.22 \cdot 10^{81}$ & $1.02 \cdot 10^{83}$ & $1.00 \cdot 10^{00}$ & $1.00 \cdot 10^{00}$ \\
\hline 3 & $1.11 \cdot 10^{71}$ & $1.14 \cdot 10^{73}$ & $1.00 \cdot 10^{00}$ & $1.00 \cdot 10^{00}$ \\
\hline 4 & $1.10 \cdot 10^{61}$ & $1.27 \cdot 10^{63}$ & $1.00 \cdot 10^{00}$ & $1.00 \cdot 10^{00}$ \\
\hline 5 & $1.14 \cdot 10^{51}$ & $1.41 \cdot 10^{53}$ & $1.00 \cdot 10^{00}$ & $1.00 \cdot 10^{00}$ \\
\hline 6 & $1.21 \cdot 10^{41}$ & $1.56 \cdot 10^{43}$ & $1.00 \cdot 10^{00}$ & $1.00 \cdot 10^{00}$ \\
\hline 7 & $1.31 \cdot 10^{31}$ & $1.74 \cdot 10^{33}$ & $1.00 \cdot 10^{00}$ & $1.00 \cdot 10^{00}$ \\
\hline 8 & $1.43 \cdot 10^{21}$ & $1.93 \cdot 10^{23}$ & $1.00 \cdot 10^{00}$ & $1.00 \cdot 10^{00}$ \\
\hline 9 & $1.58 \cdot 10^{11}$ & $2.15 \cdot 10^{13}$ & $1.00 \cdot 10^{00}$ & $1.00 \cdot 10^{00}$ \\
\hline 10 & $1.84 \cdot 10^{01}$ & $2.38 \cdot 10^{03}$ & $9.46 \cdot 10^{-01}$ & $9.46 \cdot 10^{-01}$ \\
\hline 11 & $1.00 \cdot 10^{00}$ & $2.65 \cdot 10^{-07}$ & $2.05 \cdot 10^{-09}$ & $3.88 \cdot 10^{-16}$ \\
\hline 12 & $1.00 \cdot 10^{00}$ & $2.94 \cdot 10^{-17}$ & $1.11 \cdot 10^{-10}$ & - \\
\hline
\end{tabular}

while $\left\|I-\widehat{G}^{(M)}\right\|$ from Algorithm 4.1 in Table 4.1 - Table 3.5 is always around $\mathbf{u}$, which is consistent with (127) as expected. Thus, Algorithm 4.1 gives more accurate results 
TABLE 4.4. Result for Rump matrix randmatsym(n,cnd) with $n=$ 500 , several anticipated condition numbers $\mathrm{cnd}$ and $\varepsilon_{t o l}=10^{-10}$. ( $t_{i}$ : elapsed time [sec], $m_{i}$ : number of iterations. )

\begin{tabular}{cccccccccc}
\hline & & \multicolumn{3}{c}{ Algorithm 2.8 } & & \multicolumn{3}{c}{ Algorithm 4.1 } \\
\cline { 3 - 5 } \cline { 7 - 9 } cnd & $\kappa(A)$ & $t_{1}$ & $m_{1}$ & $\left\|I-G^{\left(m_{1}\right)}\right\|$ & & $t_{2}$ & $M$ & $\left\|I-G^{(M)}\right\|$ \\
\hline $10^{20}$ & $5.76 \cdot 10^{21}$ & 1.83 & 3 & $2.78 \cdot 10^{-11}$ & & 0.96 & 3 & $4.44 \cdot 10^{-16}$ \\
$10^{40}$ & $3.32 \cdot 10^{43}$ & 4.88 & 5 & $2.78 \cdot 10^{-11}$ & & 3.55 & 5 & $4.44 \cdot 10^{-16}$ \\
$10^{60}$ & $3.26 \cdot 10^{63}$ & 10.52 & 7 & $2.78 \cdot 10^{-11}$ & & 8.39 & 7 & $4.44 \cdot 10^{-16}$ \\
$10^{80}$ & $1.10 \cdot 10^{87}$ & 22.69 & 10 & $2.78 \cdot 10^{-11}$ & & 15.29 & 9 & $4.44 \cdot 10^{-16}$ \\
$10^{100}$ & $3.18 \cdot 10^{99}$ & 28.27 & 11 & $2.78 \cdot 10^{-11}$ & & 24.55 & 11 & $4.44 \cdot 10^{-16}$ \\
\hline
\end{tabular}

than Algorithm 2.8. As can be seen, in Table 3.5, Algorithm 4.1 requires less iterations than Algorithm 2.8. The reason is that the stopping criterion (100) in Algorithm 4.1 is satisfied when $\kappa\left(\widehat{G}^{\left(m_{2}\right)}\right) \approx 1$ and the iterations finish, while Algorithm 2.8 continues one more iteration until satisfying $\left\|I-\widehat{G}^{(k)}\right\| \approx n^{2} \mathbf{u}$ even though $\kappa\left(G^{(k)}\right) \approx 1$.

Next, we observe the measured computing time for the algorithms. We again use randmatsym $(n, c n d)$. Here, $t_{1}$ and $t_{2}$ denote the computing time for Algorithm 2.8 and that for Algorithm 4.1, respectively. For reference, let $t_{0}$ and $m_{0}$ denote the computing time and the number of iterations for Algorithm 2.8 with setting $\varepsilon_{t o l}=10^{-6}$, respectively. First, we fix $n=500$ and vary anticipated condition numbers cnd from $10^{20}$ to $10^{100}$. The results are displayed in Table 4.4. As can be seen, $t_{1}$ and $t_{2}$ gradually increase according to the condition numbers, and $t_{2}$ is smaller than $t_{1}$. This is because Algorithm 2.8 needs to calculate (28) which requires $\mathcal{O}\left(m_{1}^{2} n^{3}\right)$ flops, while Algorithm 4.1 does not. Namely, in the case of $m_{1}=M$, the computational cost of Algorithm 4.1 is less than that of Algorithm 2.8. Second, we fix $c n d=10^{19}$ and vary $n$ from 500 to 5000 . The results are displayed in Table 4.5. As expected, $t_{2}$ is smaller than $t_{1}$, which is a similar result to the previous example.

It turns out that Algorithm 4.1 is faster and gives more accurate results than Algorithm 2.8. All the numerical results shown in Table 4.1 - Table 4.5 are consistent with our analysis mentioned in Subsection 4.2. 
TABLE 4.5. Result for Rump matrix randmatsym $(n$, cnd $)$ with several $n$ and $\kappa(A) \approx 10^{19}$. $\left(t_{i}\right.$ : elapsed time [sec], $m_{i}$ : number of iterations. )

\begin{tabular}{cccccccccc}
\hline & & \multicolumn{3}{c}{ Algorithm 2.8} & & \multicolumn{3}{c}{ Algorithm 4.1 } \\
\cline { 7 - 9 }$n$ & $\varepsilon_{\text {tol }}$ & $t_{1}\left(t_{0}\right)$ & $m_{1}\left(m_{0}\right)$ & $\left\|I-G^{\left(m_{1}\right)}\right\|$ & & $t_{2}$ & $M$ & $\left\|I-G^{(M)}\right\|$ \\
\hline 500 & $10^{-10}$ & $1.74(1.76)$ & $3(3)$ & $2.85 \cdot 10^{-11}$ & & 1.28 & 3 & $4.44 \cdot 10^{-16}$ \\
1000 & $10^{-9}$ & $12.03(11.96)$ & $3(3)$ & $1.57 \cdot 10^{-10}$ & & 6.53 & 3 & $6.67 \cdot 10^{-16}$ \\
2000 & $10^{-9}$ & $70.61(68.51)$ & $3(3)$ & $6.55 \cdot 10^{-10}$ & & 38.10 & 3 & $4.80 \cdot 10^{-16}$ \\
3000 & $10^{-8}$ & $225.03(221.92)$ & 3 & $(3)$ & $1.10 \cdot 10^{-09}$ & & 118.14 & 3 & $4.80 \cdot 10^{-16}$ \\
4000 & $10^{-8}$ & $849.85(495.80)$ & 4 & $(3)$ & $1.78 \cdot 10^{-09}$ & & 613.04 & 4 & $4.44 \cdot 10^{-16}$ \\
5000 & $10^{-8}$ & $1728.70(1018.03)$ & 4 & $(3)$ & $2.78 \cdot 10^{-09}$ & & 1234.72 & 4 & $9.77 \cdot 10^{-16}$ \\
\hline
\end{tabular}

REMARK 10. One may be interested in comparing Algorithm 4.1 with a standard Cholesky factorization applying long precision arithmetic. Such numerical results for Algorithm 2.8 are presented in terms of measured computing time for verifying the positive definiteness of an input matrix in Section 3. For Algorithm 4.1, we expect that similar results to those in this section are provided. 

Chapter 5

\section{CONCLUSION}


In Chapter 3, we presented detailed analysis of the algorithm for accurate and robust inverse Cholesky factorization for symmetric positive definite matrices which are extremely ill-conditioned. The presented analysis shows that if we can use high precision computations for dot product, then the condition number of a preconditioned matrix is reduced by a factor around $n^{2} \mathbf{u}$ in each iteration. Moreover, from our presented analysis, we can choose adequate computational precision for calculating dot products in the algorithm, which is sufficient for convergence and almost optimal in terms of computational effort. The numerical results are consistent with our analysis.

In Chapter 4, we propose a modified version of the Ogita-Oishi's algorithm [7] for calculating an accurate inverse of the exact Cholesky factor for ill-conditioned matrices. The presented analysis shows that the proposed algorithm (Algorithm 4.1) gives more accurate results than the original one (Algorithm 2.8). The numerical results are consistent with our analysis. Moreover, the computational cost of Algorithm 4.1 is less than that of Algorithm 2.8. Thus, Algorithm 4.1 is faster than Algorithm 2.8. 


\section{Appendix A}

\section{Executable Matlab code}


The following is executable Matlab-code for Algorithm 2.8. The code for Ozaki_Split , acc_trmm_LU, acc_trmm_LL1 and acc_trmm_LL2 are provided by Associate Professor Katsuhisa Ozaki at Shibaura Institute of Technology.

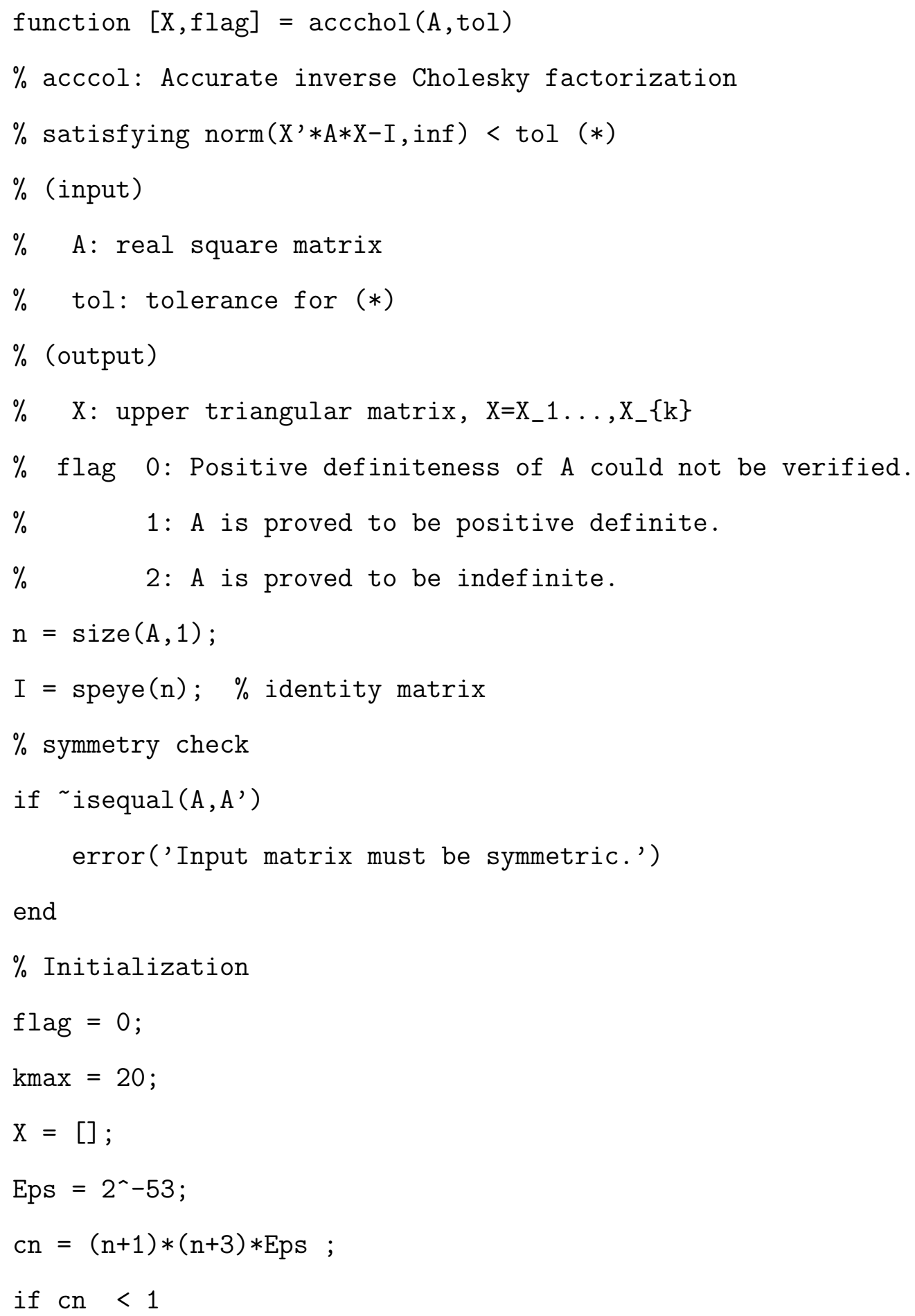




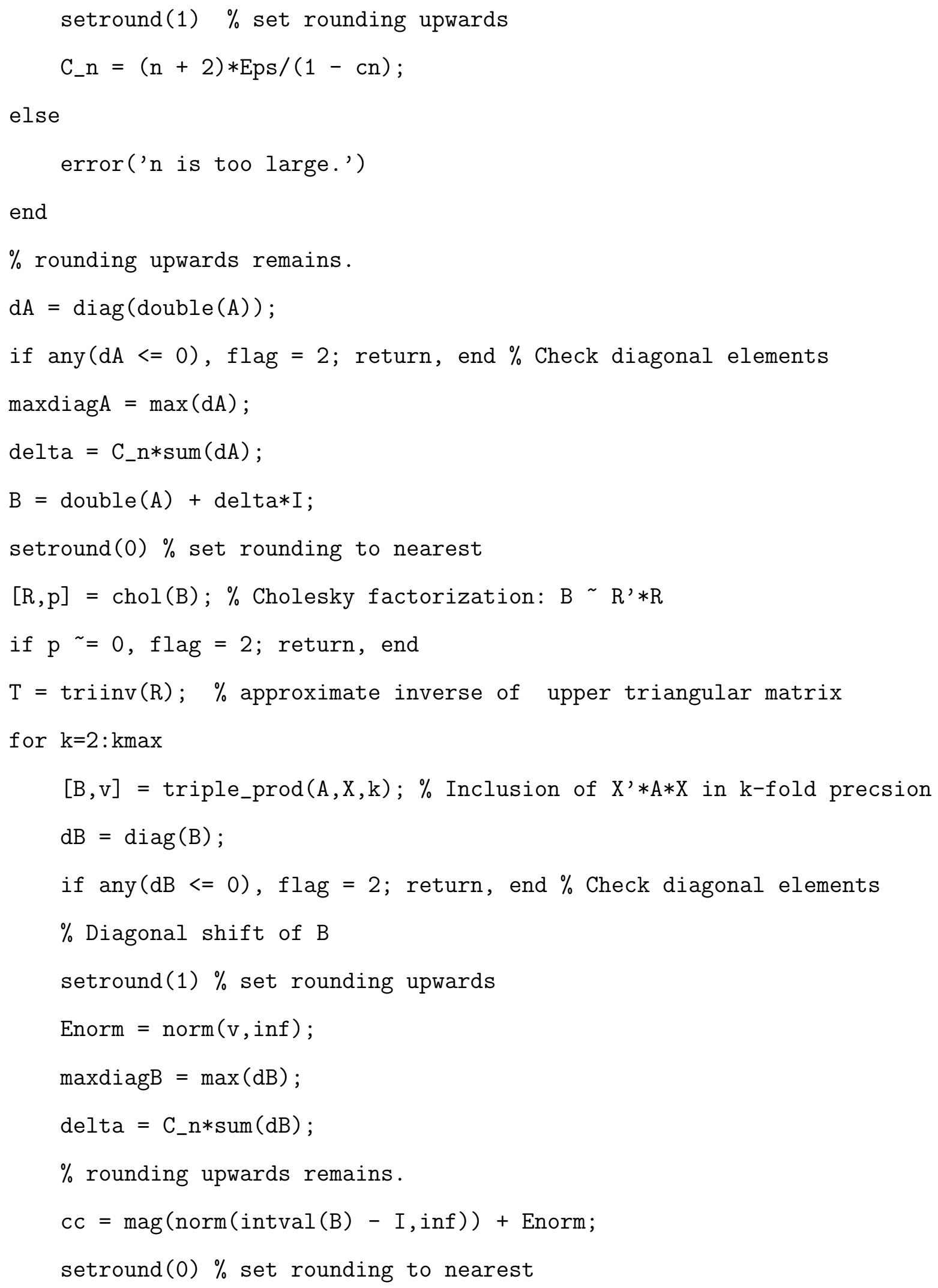




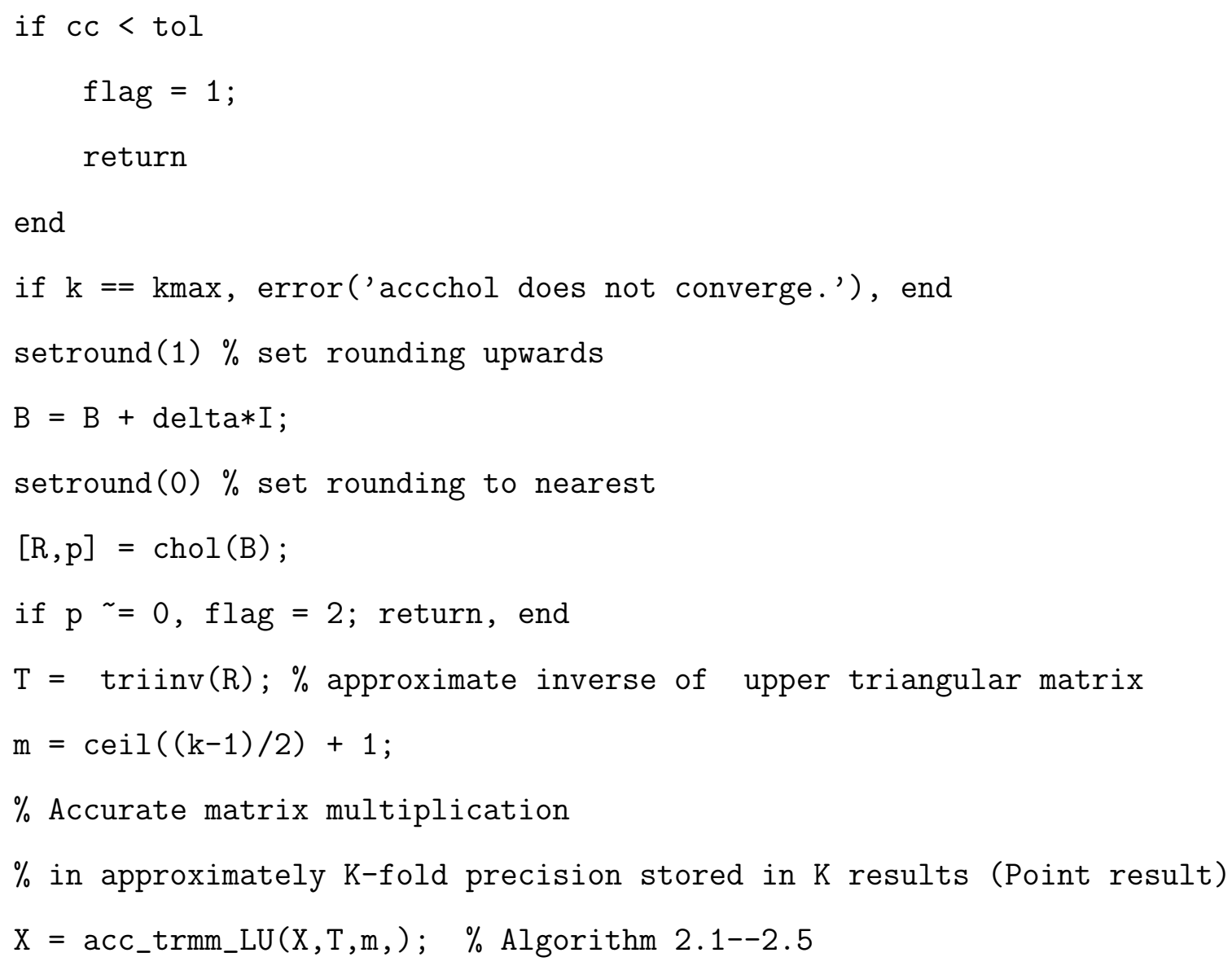




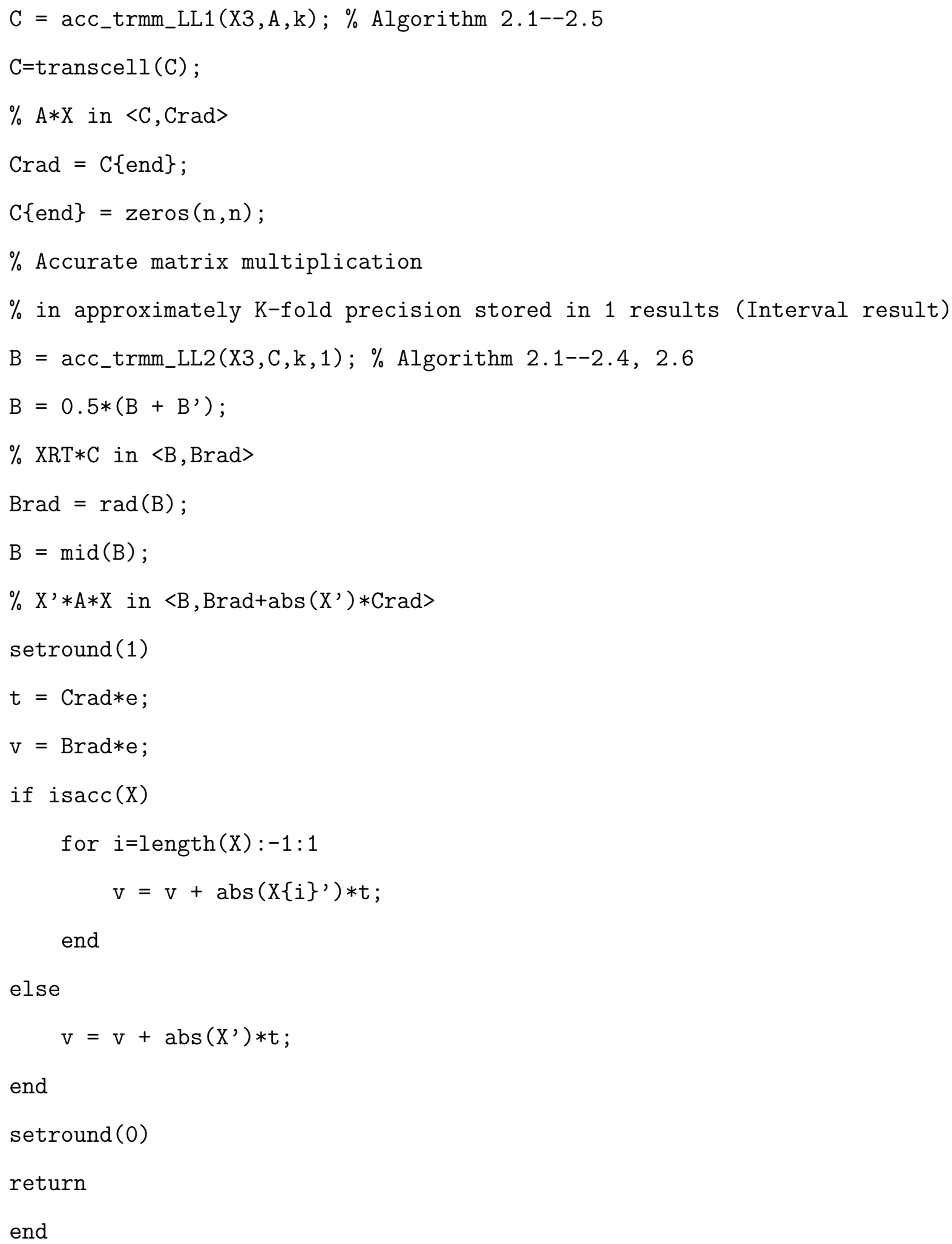


The following is executable Matlab-code for Algorithm 4.1.

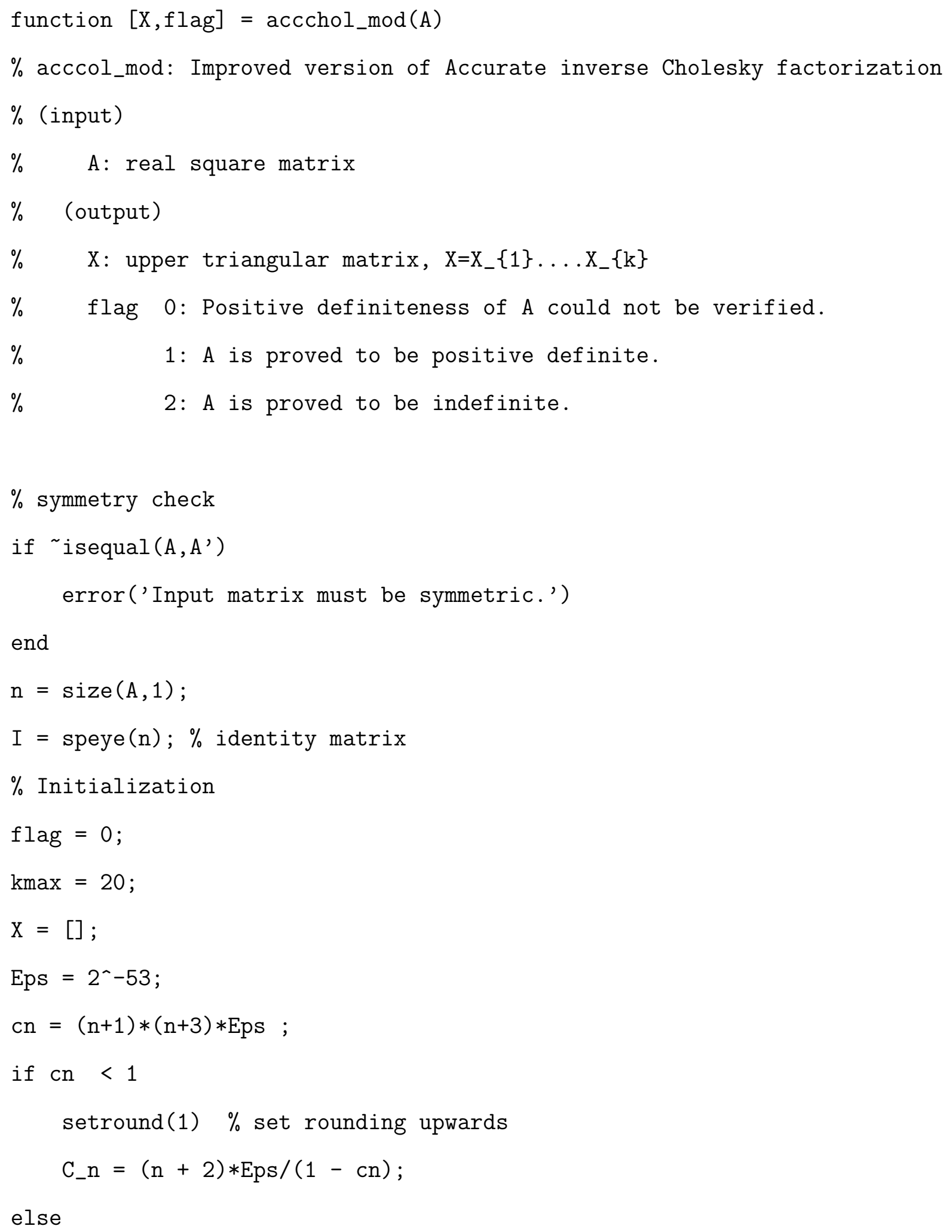


error('n is too large.')

end

$\%$ rounding upwards remains.

$\mathrm{dA}=\operatorname{diag}(\operatorname{double}(\mathrm{A}))$;

if $\operatorname{any}(\mathrm{dA}<=0)$, flag $=2$; return, end $\%$ Check diagonal elements

$\operatorname{maxdiag} A=\max (d A)$;

delta $=$ C_n $*$ sum $(d A)$;

$B=\operatorname{double}(A)+\operatorname{delta} * I$

setround (0)

$[R, p]=\operatorname{chol}(B) ; \%$ Cholesky factorization: $B \sim R^{\prime} * R$

if $\mathrm{p} \sim=0$, flag $=2$; return, end

$\mathrm{T}=\operatorname{triinv}(\mathrm{R}) ; \quad \%$ Solve $\mathrm{R} * \mathrm{X}=\mathrm{I}$.

for $k=2: k m a x$

$\%$ Inclusion of $\mathrm{X}^{\prime} * \mathrm{~A} * \mathrm{X}$ in $\mathrm{k}$-fold precsion stored 1 result

$[\mathrm{B}, \mathrm{v}]=$ triple_prod $(\mathrm{A}, \mathrm{X}, \mathrm{k})$;

$\mathrm{dB}=\operatorname{diag}(\mathrm{B})$

if $\operatorname{any}(\mathrm{dB}<=0)$, flag $=2$; return, end $\%$ Check diagonal elements setround(1)

Enorm $=$ norm $(v$, inf $)$;

$b=\operatorname{sum}(\operatorname{abs}(B-\operatorname{diag}(d B)), 2) ;$

C_n2 $=(\mathrm{n}+1) *$ Eps $/(1-2 *(\mathrm{n}+1) *$ Eps $)$;

max_error $=\max \left(\mathrm{C} \_n 2 * \operatorname{sum}(\mathrm{dB})\right.$, Enorm $)$;

setround $(-1)$

$c c=\min (d B-b)$

setround (0)

if $\quad c>$ max_error \% Stopping criterion

$[R, p]=\operatorname{chol}(B)$; 


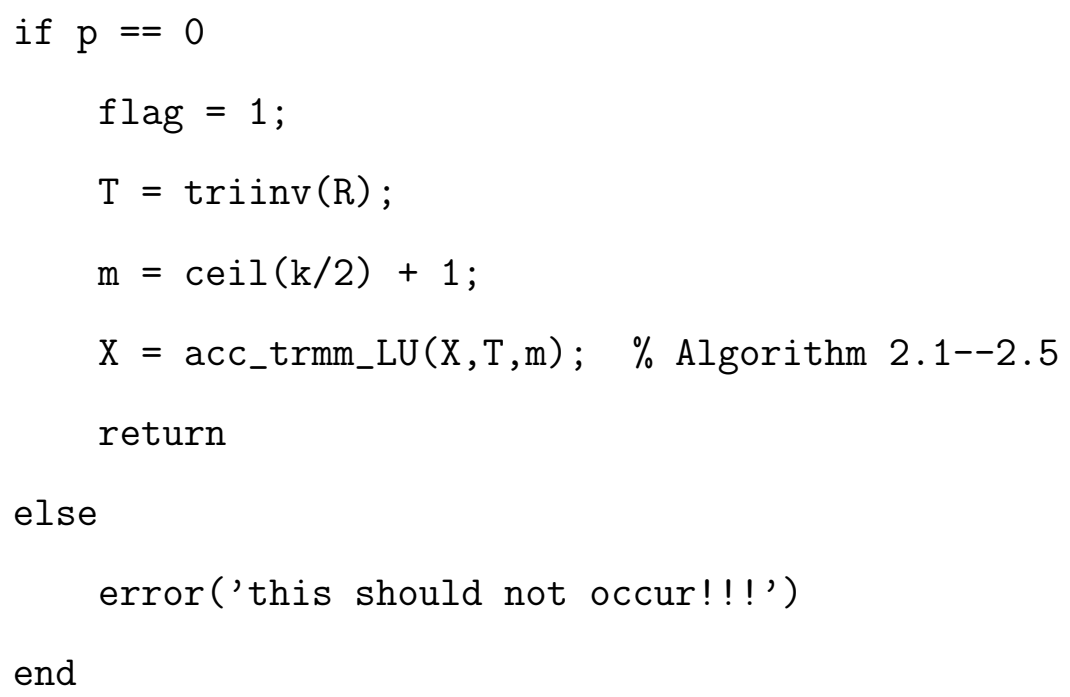


The following is executable Matlab-code for testmatrix.

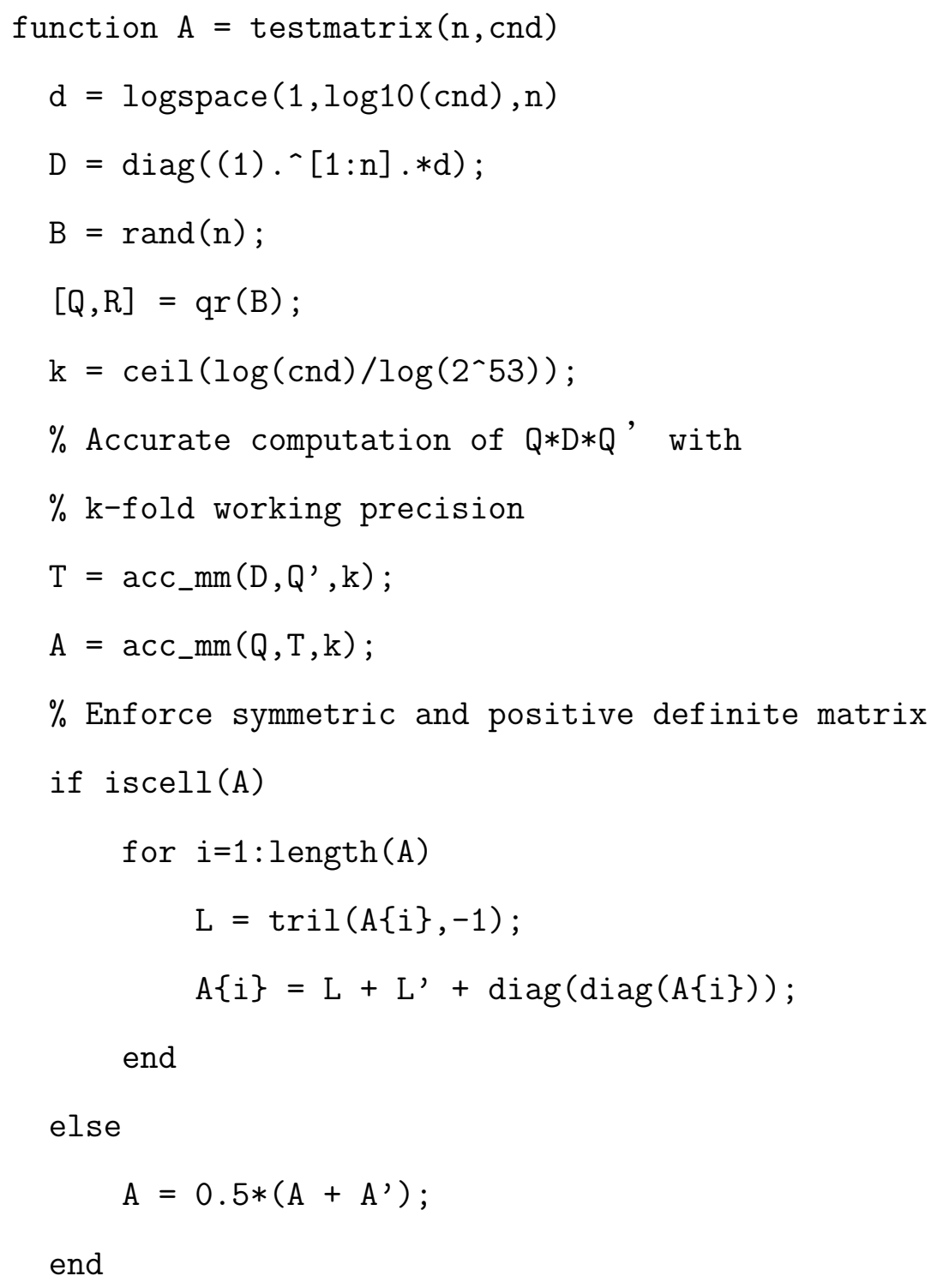


The following is executable C-code for mpfr_vchol.

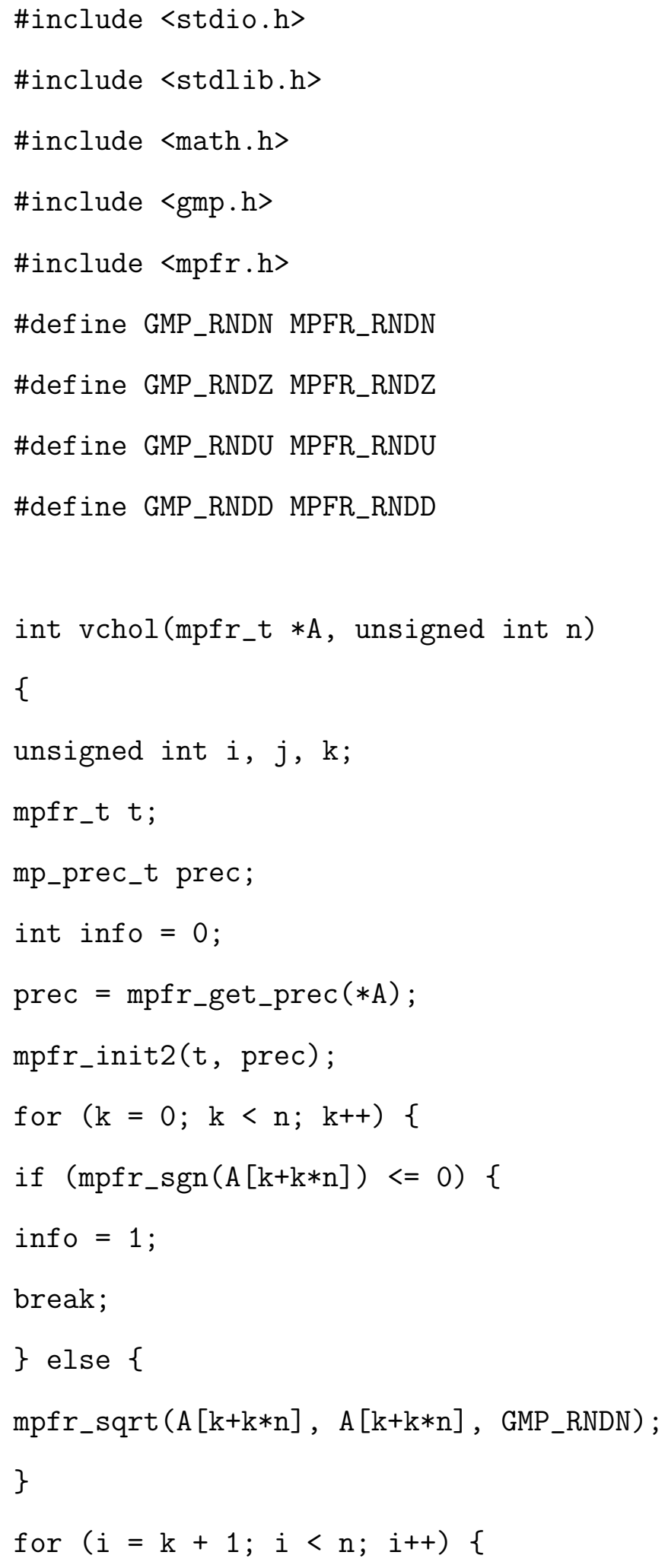




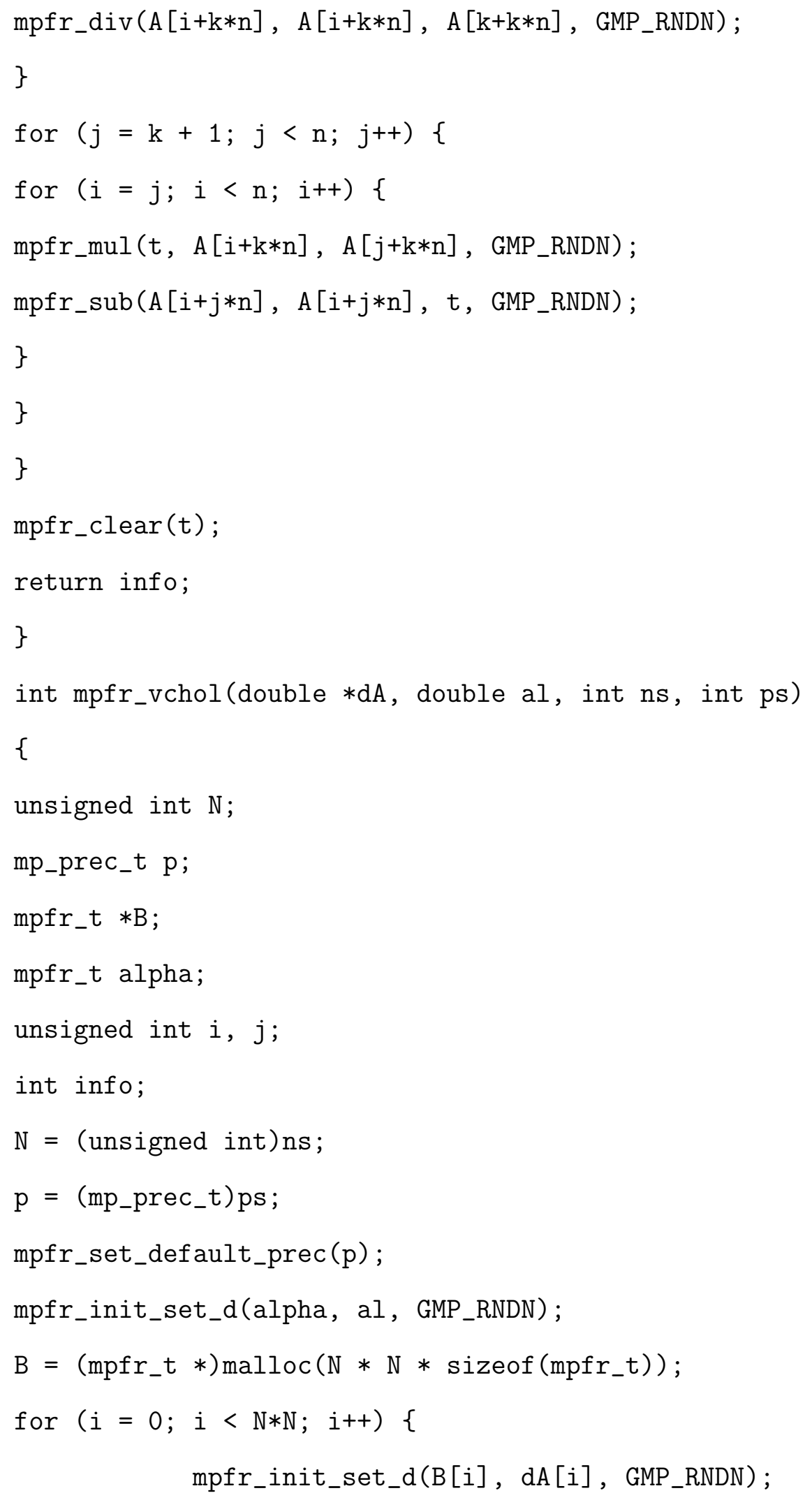




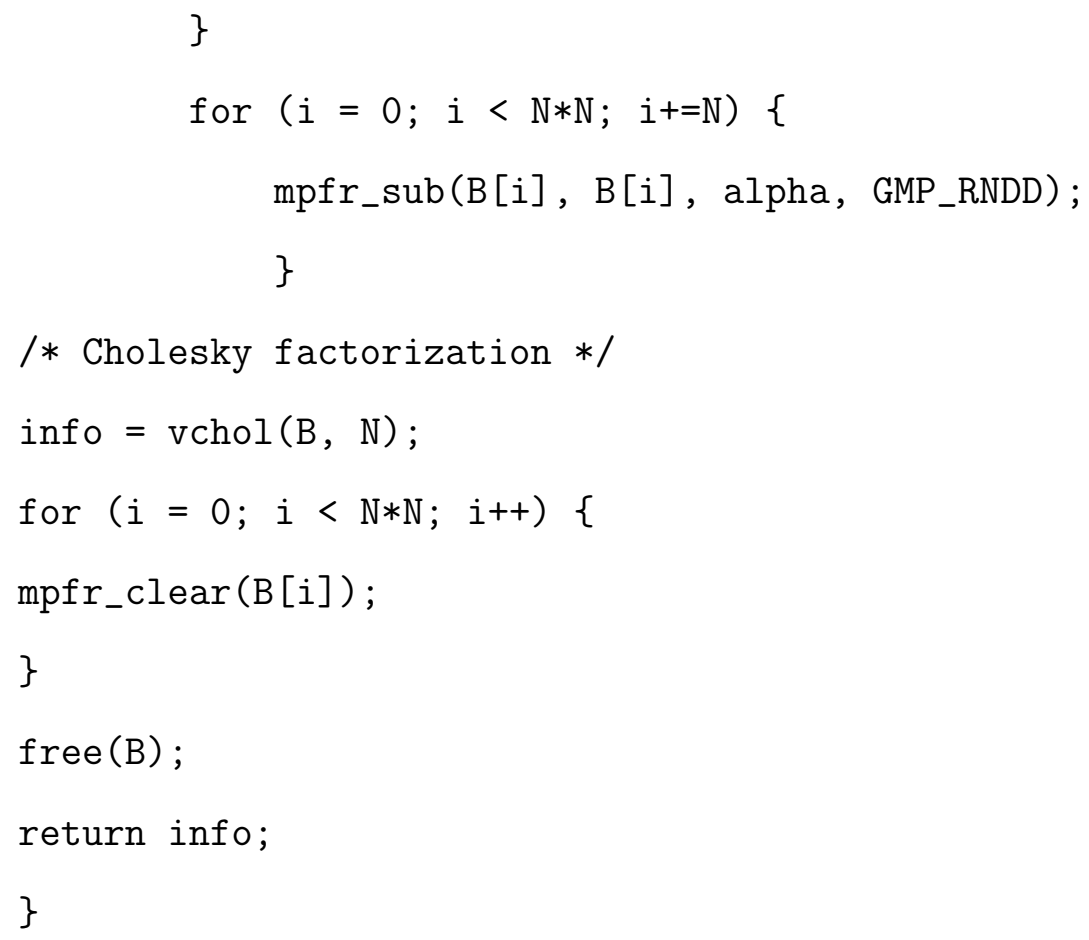




\section{BIBLIOGRAPHY}

1. J. Demmel, On floating point errors in Cholesky, LAPACK Working Note 14 CS89-87, Department of Computer Science, University of Tennessee, Knoxville, TN, USA, 1989.

2. GMP, Nonnegative matrices in the mathematical sciences, version 5.1.0, 2012., Code and documentation available at http://gmplib.org/.

3. N. J. Higham, Accuracy and Stability of Numerical Algorithms, 2nd ed., SIAM, Philadelphia, PA, 2002.

4. D. Knuth, The art of computer programming: Seminumerical algorithm vol. 2, Addison Wesley, Reading, Massachusetts, 1969.

5. MPFR, The multiple precision floating-point reliable library, version 3.1.2, March 2013., Code and documentation available at http://www.mpfr.org/.

6. T. Ogita, Accurate matrix factorization: Inverse lu and inverse qr factorizations, SIAM Journal on Matrix Analysis and Applications 31 (2010), no. 5, 2477-2497.

7. T. Ogita and S. Oishi, Accurate and robust inverse cholesky factorization, Nonlinear Theory and Its Applications, IEICE 3 (2012), 103-111.

8. T. Ogita, S. M. Rump, and S. Oishi, Accurate sum and dot product, SIAM J. Sci. Comput. 26 (2005), 1955-1988.

9. T. Ohta, T. Ogita, S. M. Rump, and S. Oishi, Numerical verification method for arbitrarily ill-conditioned linear systems, Trans. JSIAM 15 (2005), no. 3, 269-287, (in Japanese).

10. S. Oishi, K. Tanabe, T. Ogita, and S. M. Rump, Convergence of Rump's method for inverting arbitrarily ill-conditioned matrices, J. Comp. Appl. Math. 205 (2007), no. 1, 533-544.

11. K. Ozaki, T. Ogita, S. Oishi, and S. M. Rump, Error-free transformations of matrix multiplication by using fast routines of matrix multiplication and its applications, Numerical Algorithms 59 (2012), 95-118. 
12. S. M. Rump, Approximate inverses of almost singular matrices still contain useful information, Forschungsschwerpunktes Informations - und Kommunikationstechnik,, Technical Report (1990), 90.1.

13. _ A class of arbitrarily ill-conditioned floating-point matrices, SIAM J. Matrix Anal. Appl. 12 (1991), no. 4, 645-653.

14. __ INTLAB - INTerval LABoratory, Developments in Reliable Computing (Tibor Csendes, ed.), Kluwer Academic Publishers, Dordrecht, 1999, http:// www.ti3.tuhh.de/rump/, pp. 77-104.

15. _ Verification of positive definiteness, BIT Numerical Mathematics 46 (2006), 433-452.

16. Inversion of extremely ill-conditioned matrices in floating-point, Japan J. Indust. Appl. Math. 26 (2009), 249-277.

17. S. M. Rump and T. Ogita, Super-fast validated solution of linear systems, J. Comput. Appl. Math. 199 (2007), no. 2, 199-206.

18. S. M. Rump, T. Ogita, and S. Oishi, Accurate floating-point summation part I: faithful rounding, SIAM J. Sci. Comput. 31 (2008), 189-224.

19. _ Accurate floating-point summation part II: sign, K-fold faithful and rounding to nearest, SIAM J. Sci. Comput. 31 (2008), 1269-1302.

20. A. Stathopoulos and $\mathrm{K} . \mathrm{Wu}$, A block orthogonalization procedure with constant synchronization requirements, SIAM J. Sci. Comput. 23 (2002), 2165-2182.

21. G. W. Stewart and J. G. Sun, Matrix perturbation theory, Academic Press, Boston, 1990.

22. Y. Yanagisawa and T. Ogita, Convergence analysis of accurate inverse Cholesky factorization, JSIAM Letters 5 (2013), 25-28. 


\section{早稲田大学 博士（工学）学位申請 研究業績書} 氏名 柳澤 優香

\begin{tabular}{|c|c|}
\hline 種 類 別 & 発表・発行年月、 連名者（申請者含む） \\
\hline $\begin{array}{l}\text { 国際会議 } \\
\text { における } \\
\text { 口頭発表 }\end{array}$ & $\begin{array}{l}\text { [1]. Y. Yanagisawa, T. Ogita: Convergence analysis of accurate inverse Cholesky } \\
\text { factorization, JSIAM Letters, vol. } 5 \text { (2013), pp. 25-28. } \\
\text { [2]. Y. Yanagisawa, T. Ogita, S. Oishi: Convergence analysis of an algorithm for } \\
\text { accurate inverse Cholesky factorization, Japan Journal of Industrial and Applied } \\
\text { Mathematics, vol. } 31 \text { (2014), pp. 461-482. } \\
\text { [3]. Y. Yanagisawa, T. Ogita, S. Oishi: A modified algorithm for accurate inverse } \\
\text { Cholesky factorization, Nonliner Theory and Its Applications, vol. 5, no. 1 (2014), } \\
\text { pp. 35-46. } \\
\text { [4]. Y. Yamamoto, Y. Nakatsukasa, Y. Yanagisawa, T. Fukaya: Roundoff error analysis } \\
\text { of the CholeskyQR2 algorithm, Electronic Transactions on Numerical Analysis, vol. } 44 \\
\text { (2015), pp. 306-326. } \\
\text { [5] Y. Yamamoto, Y. Nakatsukasa, Y. Yanagisawa, T. Fukaya: Roundoff error analysis } \\
\text { of the CholeskyQR2 algorithm in an oblique inner product, to appear. } \\
\text { [1]. Y. Yanagisawa, T. Ogita, S. Oishi: A modified algorithm for accurate inverse } \\
\text { Cholesky factorization, 2013 International Symposium on Nonlinear Theory and its } \\
\text { Applications (NOLTA2013), Santa Fe, USA, Sep. 2013. } \\
\text { [2]. T. Fukaya, Y. Nakatsukasa, Y. Yanagisawa, Y. Yamamoto: CholeskyQR2: A Practical } \\
\text { and Communication-Avoiding Algorithm for Tal1-Skinny QR Factorizations, } \\
\text { Proceedings of the 5th Workshop on Latest Advances in Scalable Algorithms for } \\
\text { Large-Scale Systems, pp. 31-38, New Orleans Louisiana, USA, Nov. 2014. } \\
\text { [3]. T. Fukaya, Y. Nakatsukasa, Y. Yanagisawa, Y. Yamamoto: CholeskyQR2: A Practical } \\
\text { and Communication-Avoiding Algorithm for Tall-Skinny QR Factorizations, The } \\
\text { CholeskyQR2 algorithm and its applications20th ASE Seminar (Advanced } \\
\text { Supercomputing Environment), The University of Tokyo, Oct. 2015. } \\
\text { [4]. Y. Yamamoto, Y. Nakatsukasa, Y. Yanagisawa, T. Fukaya: Roundoff error analysis } \\
\text { of the CholeskyQR2: SIAM Conference on Applied Linear Algebra (SIAM LA15), Atlanta, } \\
\text { USA, Nov. 2015. } \\
\text { and Communication-Avoiding Algorithm for Tall-Skinny QR Factorizations: SIAM } \\
\text { Conference on Applied Linear Algebra (SIAM LA15), Atlanta, USA, Nov. 2015. }\end{array}$ \\
\hline
\end{tabular}




\section{早稲田大学 博士（工学） 学位申請 研究業績書}

\begin{tabular}{|c|c|}
\hline 種 類 別 & 発表・発行掲載誌名、 \\
\hline $\begin{array}{l}\text { 国内学 } \\
\text { 会・シンポ } \\
\text { ジウム等 } \\
\text { における } \\
\text { ロ頭発表 }\end{array}$ & 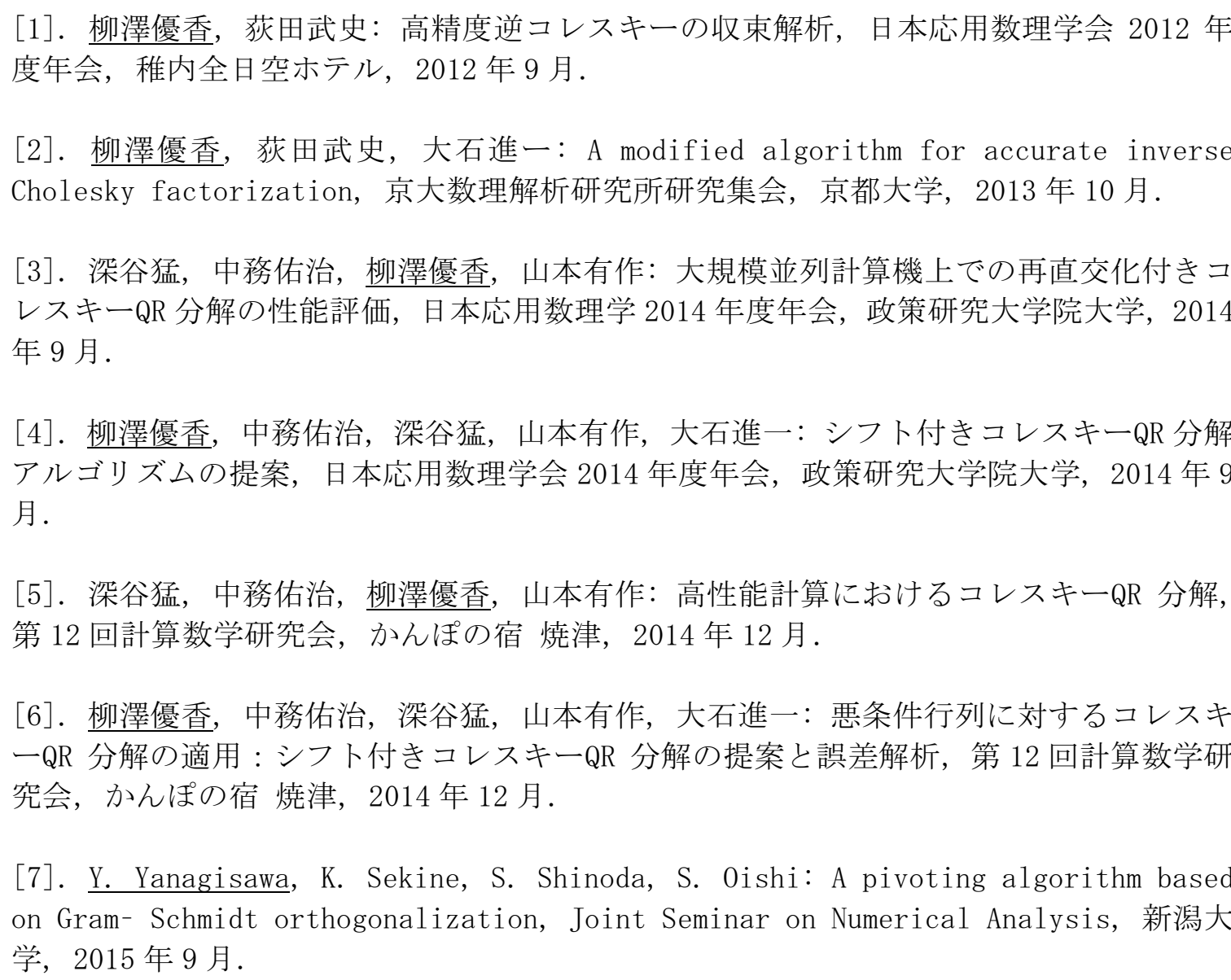 \\
\hline $\begin{array}{l}\text { 国内 学 } \\
\text { 会・シンポ } \\
\text { ジウム等 } \\
\text { における } \\
\text { ポスター } \\
\text { 発表 }\end{array}$ & $\begin{array}{l}\text { [1]. 柳澤優香, 荻田武史, 大石進一: A modified algorithm for accurate inverse } \\
\text { Cholesky factorization, 第 } 42 \text { 回数值解析シンポジウム NAS } 2013 \text {, 松山道後温泉, } 2013 \\
\text { 年 } 6 \text { 月. } \\
\text { [2]. 野田ふみ, 柳澤優香, 大石 進一：QR 分解を用いた連立一次方程式の解に対する精 } \\
\text { 度保証付き数伹計算法, 日本応用数理学会 } 2015 \text { 年度年会, 金沢大学, } 2015 \text { 年 } 9 \text { 月. } \\
\text { [3]. 深谷 猛, 中務 佑治, 柳澤 優香, 山本 有作：重み付き内積空間における行列の QR } \\
\text { 分解アルゴリズムの考察 一高性能計算の視点から, 日本応用数理学会 } 2015 \text { 年度年会, 金 } \\
\text { 沢大学, } 2015 \text { 年 } 9 \text { 月. }\end{array}$ \\
\hline
\end{tabular}

